\title{
Opérateurs de Ruelle-Mayer généralisés et analyse en moyenne des algorithmes d'Euclide et de Gauss
}

\author{
par \\ Brigitte Vallée (Caen)
}

Introduction. L'algorithme d'Euclide, étroitement lié à celui des fractions continues, est un des plus anciens algorithmes existant. Son étude emprunte trois directions relativement distinctes.

(i) La première direction, la plus ancienne, remonte à 1800. Gauss [Ga] a alors posé le problème de l'évolution de la distribution des données au cours de l'algorithme des fractions continues; c'est ce que l'on appelle maintenant l'analyse dynamique de l'algorithme : on débute l'algorithme avec une densité initiale $f$ sur $[0,1]$; quelle est la densité après $k$ itérations de l'algorithme? Gauss a conjecturé l'existence d'une densité-limite solution d'une équation fonctionnelle; il restait à prouver l'existence de cette limite et à mesurer la vitesse de convergence. Les premiers résultats dans ce sens ont été obtenus un siècle plus tard par Kuz'min [Ku] (1928) et Lévy [Le] (1929). Finalement Babenko [Ba] et Wirsing [Wi], autour de 1975, ont complètement résolu le problème en utilisant des méthodes d'analyse fonctionnelle nouvelles dans le domaine : ils ont introduit une famille particulière d'opérateurs qu'on appelle depuis les opérateurs de Ruelle-Mayer. Ces opérateurs $\mathcal{G}_{s}$ dépendent d'un paramètre $s$, et Babenko et Wirsing les utilisent en $s=2$.

(ii) La deuxième direction est celle de l'analyse de la complexité de l'algorithme, c'est-à-dire l'étude du nombre d'itérations. L'analyse de la complexité dans le pire des cas a été effectuée dès 1850 par Lamé [Lam], mais l'analyse en moyenne, c'est-à-dire l'étude de la variable aléatoire $L$ "nombre d'itérations" a seulement débuté vers 1970; les méthodes ont été très vite diverses, puisque Heilbronn et Dixon ont évalué à peu près en même temps l'espérance $E[L]$ du nombre d'itérations par deux méthodes différentes : la méthode d'Heilbronn [Hei] est purement combinatoire, tandis que celle de Dixon [Di] s'appuie sur le modèle continu associé et utilise les résultats

1991 Mathematics Subject Classification: Primary 11K; Secondary 47 and 70G. 
de Philipp. En 1994, Hensley [He] a effectué une percée en démontrant le caractère asymptotiquement gaussien de cette variable $L$; il a obtenu ce résultat en utilisant fortement les propriétés des opérateurs de Ruelle-Mayer lorsque le paramètre $s$ est proche de 2 . En simplifiant cette preuve, très technique, on obtient un résultat plus faible qui donne une preuve alternative tout à fait naturelle des résultats d'Heilbronn et Dixon [FV].

(iii) Le troisième axe relève de la théorie probabiliste des nombres; on y étudie les propriétés métriques des continuants $Q_{k}$ et en particulier la convergence de la loi des variables aléatoires $\log Q_{k}$ vers la loi normale. Les méthodes employées jusqu'à une date récente étaient essentiellement probabilistes, avec des interventions assez fortes de la théorie ergodique. C'est ainsi que Philipp [Ph], en assimilant la variable $\log Q_{k}$ à une somme de variables presque indépendantes, a obtenu en 1970 un théorème central-limite, avec une vitesse de convergence en $O\left(k^{-1 / 5}\right)$. Cette vitesse de convergence a été améliorée depuis par Misyavichyus [Mi] (1987), jusqu'en $O(\log k / \sqrt{k})$. Très récemment, Morita [Mo] (1994) démontre un théorème central-limite dans un cadre général en utilisant les opérateurs de Ruelle liés aux systèmes dynamiques. En particularisant ensuite les opérateurs - qui deviennent alors les opérateurs de Ruelle-Mayer - et en introduisant la même approximation que celle de Philipp sur les variables $\log Q_{k}$, il atteint la borne conjecturée sur la vitesse de convergence, égale à $O(1 / \sqrt{k})$. Cependant, la généralité de la méthode et l'approximation faite sur les variables ne lui permettent ni de préciser d'autres phénomèmes caractérisant le comportement asymptotique des variables $\log Q_{k}$, ni de travailler avec une densité quelconque.

Ainsi, un des problèmes non complètement résolus autour de l'algorithme d'Euclide est du troisième type (iii). C'est aussi la seule direction où l'on n'a pas cherché à utiliser directement les opérateurs de Ruelle-Mayer. Le premier axe de ce travail consiste donc à utiliser directement les opérateurs de Ruelle-Mayer pour l'étude de la loi limite de $\log Q_{k}$, et ce, dans le cas d'une densité quelconque : cette utilisation directe permettra de travailler effectivement avec la variable $\log Q_{k}$ sans recours à des approximations.

L'algorithme de Gauss, qui effectue la réduction des réseaux de dimension 2 , se présente, dans un modèle complexe adapté, comme la généralisation formelle et naturelle de l'algorithme des fractions continues [DFV]. L'analyse de la complexité de l'algorithme de Gauss est importante, car cet algorithme sert comme procédure essentielle dans tous les algorithmes de réduction des réseaux de dimension supérieure. Par ailleurs, la réduction des réseaux est extrêmement utile dans des domaines très divers, tels la programmation linéaire en nombres entiers, la factorisation des polynômes à coefficients entiers ou la cryptographie. L'algorithme LLL [LLL], qui est le plus célèbre parmi les algorithmes de réduction, a un comportement algorithmique encore mal compris, malgré quelques résultats partiels [DV], et 
toute entreprise d'analyse de complexité de cet algorithme doit bien sûr commencer par l'analyse de l'algorithme de Gauss : analyse dans le pire des cas, analyse en moyenne. On peut chercher à effectuer dans le même temps l'analyse dynamique de l'algorithme, intéressante en elle-même et susceptible de conséquences importantes sur l'analyse en moyenne.

La complexité dans le pire des cas a été étudiée de manière précise [Lag], [Va1], [KS] : on démontre que c'est essentiellement la même que celle de l'algorithme d'Euclide. Vis à vis des deux autres analyses - analyse en moyenne, analyse dynamique -, les comportements des deux algorithmes sont vraiment différents. Ces deux analyses ont déjà été effectuées, lorsque la densité initiale est uniforme, dans [DFV]; les méthodes d'analyse fonctionnelle employées font intervenir la famille d'opérateurs de Ruelle-Mayer déjà utilisée dans l'algorithme d'Euclide; on utilise alors ces opérateurs $\mathcal{G}_{s}$ pour $s=4$.

Le deuxième axe de ce travail consiste ainsi à effectuer l'analyse en moyenne et l'analyse dynamique de l'algorithme de Gauss, dans le cas d'une densité quelconque, à l'aide de méthodes d'analyse fonctionnelle similaires à celles déjà utilisées.

Pourtant, dans le cas où la densité initiale n'est plus uniforme, les opérateurs de Ruelle-Mayer classiques ne paraissent pas adaptés, dans aucune des deux problématiques. On introduit donc une nouvelle famille d'opérateurs, appelée opérateurs de Ruelle-Mayer généralisés, qui permettent de traiter le cas général d'une densité quelconque.

Ces opérateurs opèrent sur des fonctions holomorphes à deux variables. Contrairement aux opérateurs classiques de Ruelle-Mayer, étudiés de manière extensive par Mayer [Ma1], [Ma4], [MR], les opérateurs à deux variables semblent nouveaux. Même si on peut les étudier en utilisant des résultats plus généraux dûs également à Mayer [Ma2], [Ma3], nous adoptons ici un autre point de vue : nous les étudions en liaison avec les opérateurs usuels. En particulier, nous relions les propriétés spectrales dominantes des opérateurs généralisés à celles des opérateurs usuels et nous montrons qu'il y a un héritage très fort des propriétés spectrales dominantes.

Les résultats obtenus sont les suivants :

(i) En ce qui concerne l'algorithme des fractions continues, nous étudions directement la variable aléatoire $\log Q_{k}$ (où $Q_{k}$ est le continuant). Nous démontrons la convergence de la loi de $\log Q_{k}$ vers la loi normale avec un reste en $O(1 / \sqrt{k})$, et ce pour une densité analytique quelconque. Nous décrivons aussi le comportement asymptotique de l'espérance et de la variance de $\log Q_{k}$, qui fait intervenir les propriétés spectrales dominantes et sous-dominantes des opérateurs au voisinage de $s=2$, et nous précisons la manière dont l'espérance et la variance dépendent de la densité considérée. 
Nous étudions également la convergence vers la loi normale de la variable $\log \left(Q_{k}+y Q_{k-1}\right)$ et nous obtenons, pour elle, essentiellement le même type de résultats. La méthode est fondée sur l'utilisation de la série génératrice des moments et permet d'obtenir aussi des résultats de grandes déviations.

(ii) Dans l'étude de l'algorithme de Gauss, nous prouvons les deux résultats suivants, valables pour une classe très générale de densités :

- le nombre d'itérations de l'algorithme possède une distribution asymptotiquement géométrique,

- la dynamique de l'algorithme tend vers une configuration limite. Ce dernier résultat constitue, pour l'algorithme de Gauss, un résultat analogue à celui de Gauss-Kuz'min-Lévy pour l'algorithme des fractions continues.

Ces deux derniers résultats sont de même type que les résultats précédemment obtenus dans [DFV] dans le cas d'une densité uniforme. Contrairement à ceux-ci, qui utilisaient les comportements spectraux dominants des opérateurs en $s=4$, les résultats de ce travail dépendent de la "valuation" de la densité initiale : pour une densité initiale de valuation $r$, ce sont les propriétés spectrales des opérateurs en $s=4+2 r$ qui interviennent. Cette étude de l'algorithme de Gauss, qui est une généralisation 2-dimensionnelle de l'algorithme des fractions continues, est à comparer avec d'autres études concernant des généralisations différentes de l'algorithme des fractions continues, telle l'étude de Broise $[\mathrm{Br}]$ concernant l'algorithme de Jacobi-Perron ou celles de Mayer [Ma5]. Signalons aussi une autre généralisation 2-dimensionnelle de l'algorithme des fractions continues utilisée dans la détermination du signe du déterminant; l'algorithme correspondant a déjà été analysé [Va2] pour une classe plus restreinte de densités où le recours aux opérateurs de Ruelle-Mayer usuels est suffisant.

Le travail comprend trois parties; dans la première partie, nous définissons les opérateurs de Ruelle-Mayer généralisés et nous montrons comment ils interviennent naturellement dans les problèmes posés. Ensuite, nous étudions les propriétés spectrales de ces opérateurs généralisés (section 3), en référence constante avec les propriétés analogues des opérateurs classiques rappelées en section 2. Enfin, en utilisant les trois premières sections, nous obtenons les résultats énoncés, d'abord pour l'algorithme d'Euclide (section 4) et enfin pour l'algorithme de Gauss (section 5). Une version abrégée de ces résultats est parue dans les CRAS, novembre 1995. Il existe aussi un article général $[\mathrm{FV}]$ qui décrit le rôle des opérateurs de Ruelle-Mayer usuels dans l'analyse des algorithmes liés aux fractions continues.

1. Opérateurs de Ruelle-Mayer et analyse en moyenne. Les opérateurs de Ruelle-Mayer sont liés de manière étroite à l'opérateur de décalage des fractions continues, et servent à l'étudier. On en rappelle les 
propriétés formelles, puis on introduit les opérateurs généralisés. On montre ensuite comment ces opérateurs interviennent dans l'expression des trois objets ici étudiés : (i) la série génératrice des moments de la variable $\log Q_{k}$ pour l'algorithme des fractions continues, (ii) la distribution du nombre d'itérations de l'algorithme de Gauss, (iii) la densité des données après $k$ itérations de l'algorithme de Gauss.

1.1. Opérateur des fractions continues et continuants. L'opérateur de décalage $U$ des fractions continues est défini pour un réel $x$ de $\mathcal{I}=] 0,1[$ par

$$
U(x)=1 / x-[1 / x]
$$

où $[x]$ désigne la partie entière du réel $x$. Cet opérateur est à la base de l'algorithme des fractions continues :

Entrée : un réel $x$ de $\mathcal{I}$

Tant que $x \neq 0$ faire $x:=U(x)$.

A un réel $x$, on associe la suite $x_{0}=x, x_{1}, x_{2}, \ldots, x_{k}, \ldots$ des itérés de $x$. Si le $k$-ème itéré existe, l'algorithme des fractions continues construit sur l'entrée $x_{0}$ un développement en fraction continue

$$
x_{0}=\frac{1}{m_{1}+\frac{1}{m_{2}+\frac{1}{\frac{\ddots}{m_{k}+x_{k}}}}},
$$

où les entiers $m_{j}$ sont supérieurs ou égaux à 1 . La relation $x_{0}=h\left(x_{k}\right)$ définit une homographie de hauteur $k$, associée à un $k$-uplet $\mathbf{m}=\left(m_{1}, \ldots, m_{k}\right)$ d'entiers $m_{i} \geq 1$,

$$
h_{\mathbf{m}}(z)=h_{m_{1}, m_{2}, \ldots, m_{k}}(z)=\frac{1}{m_{1}+\frac{1}{m_{2}+\frac{1}{\frac{\ddots}{m_{k}+z}}}} .
$$

Toutes ces homographies de hauteur $k$ constituent ainsi toutes les branches inverses possibles du $k$-ème itéré de $U$. Une telle homographie $h$, de hauteur $k$, s'exprime alors à l'aide des continuants

$$
h_{\mathbf{m}}(z)=\frac{P_{k}+z P_{k-1}}{Q_{k}+z Q_{k-1}},
$$

où

$$
\begin{aligned}
Q_{k} & =Q_{k}\left(m_{1}, \ldots, m_{k}\right), & Q_{k-1} & =Q_{k-1}\left(m_{1}, \ldots, m_{k-1}\right), \\
P_{k} & =Q_{k-1}\left(m_{2}, \ldots, m_{k}\right), & P_{k-1} & =Q_{k-2}\left(m_{2}, \ldots, m_{k-1}\right) .
\end{aligned}
$$


Les polynômes continuants sont définis par récurrence

(3) $Q_{k}\left(m_{1}, \ldots, m_{k}\right)=m_{k} Q_{k-1}\left(m_{1}, \ldots, m_{k-1}\right)+Q_{k-2}\left(m_{1}, \ldots, m_{k-2}\right)$, avec $Q_{0}=1, Q_{1}\left(m_{1}\right)=m_{1}$. Il est bien connu [RS] que le polynôme continuant $Q_{k}(\mathbf{m})$ est aussi la somme de tous les monômes obtenus en barrant deux variables consécutives $m_{i} m_{i+1}$ dans le produit $m_{1} m_{2} \ldots m_{k}$. Les continuants vérifient une propriété de symétrie

$$
Q_{k}\left(m_{1}, \ldots, m_{k}\right)=Q_{k}\left(m_{k}, \ldots, m_{1}\right),
$$

et l'identité du déterminant

$$
Q_{k} P_{k-1}-Q_{k-1} P_{k}=(-1)^{k} .
$$

Pour une homographie $h$ de hauteur $k$, le transformé par $h$ du segment $\mathcal{I}$ est appelé intervalle fondamental de rang $k$. L'intervalle $\mathcal{I}$ lui-même est, à un ensemble de rationnels près, réunion disjointe de tous les intervalles fondamentaux de rang $k$ :

$$
\mathcal{I} \approx \bigcup_{|h|=k} h(\mathcal{I}) \quad \text { pour chaque } k \geq 0 .
$$

L'intervalle fondamental $h(\mathcal{I})$ s'exprime en fonction des continuants :

$$
h(\mathcal{I})=] \frac{P_{k}}{Q_{k}}, \frac{P_{k-1}+P_{k}}{Q_{k-1}+Q_{k}}[
$$

(les bornes de l'intervalle étant ordonnées ou non suivant la parité de $k$ ) et est de longueur égale à

$$
|h(\mathcal{I})|=\frac{1}{Q_{k}\left(Q_{k}+Q_{k-1}\right)} .
$$

1.2. Opérateurs usuels. Les opérateurs de Ruelle-Mayer usuels $\mathcal{G}_{s}$ servent à "inverser" l'opérateur de décalage $U$ des fractions continues. Ils sont définis pour un complexe $s$ vérifiant $\Re(s)>1$ par la relation

$$
\mathcal{G}_{s}[f](z)=\sum_{m \geq 1} \frac{1}{(m+z)^{s}} f\left(\frac{1}{m+z}\right) .
$$

Les itérés d'ordre $k$ de ces opérateurs engendrent alors les continuants d'ordre $k$ dans le sens suivant

$$
\mathcal{G}_{s}^{k}[f](z)=\sum_{m_{1} \ldots m_{k}} \frac{1}{\left(Q_{k-1} z+Q_{k}\right)^{s}} f\left(\frac{P_{k-1} z+P_{k}}{Q_{k-1} z+Q_{k}}\right),
$$

et, en particulier

$$
\mathcal{G}_{s}^{k}[f](0)=\sum_{m_{1} \ldots m_{k}} \frac{1}{Q_{k}^{s}} f\left(\frac{P_{k}}{Q_{k}}\right)=\sum_{m_{1} \ldots m_{k}} \frac{1}{Q_{k}^{s}} f\left(\frac{Q_{k-1}}{Q_{k}}\right),
$$


la seconde égalité étant due aux propriétés de symétrie (4). Une homographie $h$ de hauteur $k$ a une dérivée qui, sur le segment $\mathcal{I}$, et grâce à (5), a le signe de $(-1)^{k}$. Cela justifie l'introduction de la fonction $\widetilde{h}$ qui est l'unique fonction holomorphe qui coïncide avec

$$
\sqrt{\left|h^{\prime}(y)\right|}=\sqrt{(-1)^{k} h^{\prime}(y)}=\frac{1}{Q_{k-1} y+Q_{k}}
$$

sur le segment $\mathcal{I}$. On obtient ainsi un opérateur $\mathcal{C}_{s}^{(h)}$ défini par

$$
\mathcal{C}_{s}^{(h)}[f](z)=\widetilde{h}(z)^{s} f \circ h(z),
$$

et une forme alternative pour $\mathcal{G}_{s}$ et ses itérés, comme une somme de tels opérateurs :

$$
\mathcal{G}_{s}^{k}=\sum_{|h|=k} \mathcal{C}_{s}^{(h)}
$$

où la somme est étendue à toutes les homographies $h$ de hauteur $k$.

1.3. Introduction des opérateurs de Ruelle-Mayer généralisés. Ils dépendent de deux paramètres $s$ et $t$, vérifiant $\Re(s+t)>1$, opèrent sur des fonctions $F$ holomorphes de deux variables et sont définis formellement par

$$
\mathbf{G}_{s, t}[F](u, v)=\sum_{m \geq 1} \frac{1}{(m+u)^{s}(m+v)^{t}} F\left(\frac{1}{m+u}, \frac{1}{m+v}\right) .
$$

Leurs itérés vérifient

$$
\mathbf{G}_{s, t}^{k}[F](u, v)=\sum_{|h|=k} \widetilde{h}(u)^{s} \widetilde{h}(v)^{t} F(h(u), h(v)),
$$

où, comme précédemment, la somme est étendue aux homographies $h$ de hauteur $k$ et $\widetilde{h}$ définie comme en (9). Ainsi, l'opérateur $\mathbf{G}_{s, t}$ et ses itérés s'écrivent comme une somme d'opérateurs

$$
\mathbf{G}_{s, t}^{k}=\sum_{|h|=k} \mathbf{C}_{s, t}^{(h)}
$$

où les opérateurs $\mathbf{C}_{s, t}^{(h)}$ sont définis par

$$
\mathbf{C}_{s, t}^{(h)}[F](u, v)=\widetilde{h}(u)^{s} \widetilde{h}(v)^{t} F(h(u), h(v)) .
$$

Les opérateurs $\mathbf{G}_{s, t}$ généralisent les opérateurs usuels $\mathcal{G}_{s}$ en deux sens différents. D'une part, la restriction à la "diagonale" $u=v$ permet de retrouver les $\mathcal{C}_{s+t}$ à partir des $\mathbf{C}_{s, t}$ et donc les $\mathcal{G}_{s+t}$ à partir des $\mathbf{G}_{s, t}$ dans le sens suivant : 


$$
\mathbf{C}_{s, t}^{k}[F](u, u)=\mathcal{C}_{s+t}^{k}[f](u), \quad \mathbf{G}_{s, t}^{k}[F](u, u)=\mathcal{G}_{s+t}^{k}[f](u),
$$

où $f$ est l'application diagonale associée à $F$ par la relation $f(u)=F(u, u)$. D'autre part, lorsque $\mathbf{G}_{s, t}$ est appliqué à la fonction constante 1, il s'exprime également en fonction de $\mathcal{G}_{s+t}$,

$$
\mathbf{G}_{s, t}^{k}[1](u, v)=\mathcal{G}_{s+t}^{k}\left[\nu_{u, v}^{(s, t)}\right](0),
$$

à l'aide de la fonction $\nu_{u, v}^{(s, t)}$ qui dépend des paramètres $u$ et $v$,

$$
\nu_{u, v}^{(s, t)}(z)=\frac{1}{(1+u z)^{s}(1+v z)^{t}},
$$

comme on le voit aisément en utilisant l'égalité (8). Cette dernière expression (13) est d'ailleurs largement utilisée dans [DFV].

1.4. Les opérateurs de Ruelle-Mayer et la loi-limite de $\log Q_{k}$. La variable aléatoire $Q_{k}$ joue un rôle fondamental dans l'étude de l'algorithme des fractions continues. Pour un réel $x=x_{0}$ de l'intervalle $\mathcal{I}$, on considère le $k$-ème itéré $x_{k}=U^{k}\left(x_{0}\right)$, s'il existe, et l'homographie $h$ de hauteur $k$ vérifiant $x_{0}=h\left(x_{k}\right)$, associée à un $k$-uplet $\mathbf{m}=\left(m_{1}, \ldots, m_{k}\right)$ par $(2)$. On pose alors, avec un abus de notation habituel,

$$
Q_{k}(x):=Q_{k}\left(m_{1}, \ldots, m_{k}\right) .
$$

La variable $Q_{k}$ représente ainsi le dénominateur de la $k$-ème réduite du réel $x$; elle est définie presque partout et est constante sur chaque intervalle fondamental de rang $k$ : c'est le continuant associé à l'intervalle fondamental, et il est lié de manière étroite à la longueur de l'intervalle fondamental (7).

La proposition suivante constitue le fondement du premier axe de ce travail : elle relie, de manière très simple et nouvelle, la série génératrice des moments de la variable $Q_{k}$ et les opérateurs de Ruelle-Mayer généralisés, ou non, pour des paramètres pris au voisinage de $s=2$.

Proposition 1. La série génératrice des moments de la variable $\log Q_{k}$,

$$
M_{k}(s):=E\left[\exp \left(s \log Q_{k}\right)\right]=E\left[Q_{k}^{s}\right]
$$

s'exprime en fonction des k-èmes itérés des opérateurs de Ruelle-Mayer:

(i) lorsque la densité sur $\mathcal{I}$ est uniforme, en fonction des opérateurs usuels,

$$
M_{k}(s)=\mathcal{G}_{2-s}^{k}\left[\frac{1}{1+u}\right](0)
$$


(ii) pour une densité $f$ quelconque intégrable sur $\mathcal{I}$, en fonction des opérateurs généralisés,

$$
M_{k}[f](s)=\int_{0}^{1} \mathbf{G}_{2,-s}^{k}[F](x, 0) d x,
$$

où $F$ est définie à partir de $f$ par la relation $F(u, v)=f(u)$.

Pre u ve. La variable $Q_{k}$ est constante sur chaque intervalle fondamental de rang $k$; les intervalles fondamentaux de rang $k$ forment, à un ensemble de rationnels près, une partition de $\mathcal{I}(6)$.

Dans le cas de la densité uniforme, on utilise l'expression de la longueur de cet intervalle (7) et on obtient

$$
\begin{aligned}
M_{k}(s) & =E\left[Q_{k}^{s}\right]=\sum_{m_{1} \ldots m_{k}} Q_{k}^{s} \frac{1}{Q_{k}\left(Q_{k}+Q_{k-1}\right)} \\
& =\sum_{m_{1} \ldots m_{k}} \frac{1}{Q_{k}^{2-s}} \cdot \frac{1}{1+Q_{k-1} / Q_{k}},
\end{aligned}
$$

ce qui équivaut, grâce à (8), à la forme proposée.

Dans le cas d'une densité $f$, avec la notation définie en (9), on obtient

$$
M_{k}(s)=E\left[Q_{k}^{s}\right]=\sum_{|h|=k} \widetilde{h}(0)^{-s} \int_{h(\mathcal{I})} f(u) d u,
$$

qui se transforme par le changement de variables $h(v)=u$ effectué dans chaque intervalle fondamental,

$$
M_{k}(s)=\sum_{|h|=k} \widetilde{h}(0)^{-s} \int_{\mathcal{I}} \widetilde{h}(v)^{2} f \circ h(v) d v .
$$

L'interversion des sommations conduit alors à l'expression proposée.

R e mar que. Soit $y$ un réel de l'intervalle [0,1]. La variable $Q_{k}+y Q_{k-1}$ est également intéressante, car elle contient des informations sur la dépendance entre les variables $Q_{k}$ et $Q_{k-1}$. La série génératrice de cette variable aléatoire admet, pour une densité $f$ quelconque, l'expression plus générale

$$
M_{k}[f, y](s):=E\left[\exp \left(s \log \left(Q_{k}+y Q_{k-1}\right)\right)\right]=\int_{0}^{1} \mathbf{G}_{2,-s}^{k}[F](x, y) d x,
$$

où $F$ est encore définie à partir de $f$ par la relation $F(u, v)=f(u)$.

1.5. Opérateurs de Ruelle-Mayer et analyse en moyenne de l'algorithme de Gauss. L'algorithme de Gauss est un algorithme de réduction des réseaux en dimension 2. A partir d'une base quelconque d'un réseau de dimension 2, il construit une base minimale. Dans un modèle complexe adapté, il se présente comme une généralisation formelle de l'algorithme 
d'Euclide $[\mathrm{DFV}]$ : il utilise une extension de l'opérateur de décalage $U$ réel des fractions continues défini en (1) en un opérateur complexe

$$
U(z)=(1 / z)-[\Re(1 / z)] .
$$

Cet opérateur est ainsi le composé d'une inversion-symétrie et d'une translation entière. Appliqué à un complexe $z$ situé à l'intérieur du disque $\mathcal{D}$ de diamètre $\mathcal{I}=10,1[$, l'inversion le "sort" du disque et la translation le "ramène" dans la bande $\mathcal{B}=\{z \mid 0 \leq \Re(z)<1\}$. L'algorithme de Gauss se présente alors comme une suite d'applications de l'opérateur $U$.

Entrée : un complexe $z$ de $\mathcal{D}$

Tant que $z \in \mathcal{D}$ faire $z:=U(z)$.

Sortie : un complexe $z$ de $\mathcal{B} \backslash \mathcal{D}$.

L'analyse en moyenne de l'algorithme consiste à étudier la variable aléatoire $L$ "nombre d'itérations", lorsque les entrées $z$ sont distribuées dans $\mathcal{D}$ suivant une densité initiale $f$. Il s'agit en particulier d'étudier le comportement asymptotique de la distribution de la variable $L$ définie par la suite

$$
\varrho_{k}=\operatorname{Pr}[L \geq k+1] .
$$

Mais il est aussi fondamental de faire l'analyse "dynamique" de l'algorithme : si $F_{0}=f$ désigne la densité initiale des entrées dans le disque $\mathcal{D}$, on introduit la densité $\widetilde{F}_{k}$ qui s'établit sur $\mathcal{D}$ après $k$ itérations de l'algorithme, et il s'agit d'étudier le comportement asymptotique de la suite $\widetilde{F}_{k}$. C'est l'analogue du problème qu'avait posé Gauss pour l'algorithme d'Euclide. Contrairement à l'algorithme d'Euclide, l'algorithme de Gauss est un algorithme qui termine au bout d'un nombre fini d'itérations, sauf sur l'ensemble de mesure nulle constitué par les réels irrationnels. Ainsi la densité $\widetilde{F}_{k}$ tend nécessairement vers 0 en tout point du disque $\mathcal{D}$, car les données non réelles finissent toujours par "sortir" du disque $\mathcal{D}$. C'est donc la densité "conditionnelle" limitée au disque $\mathcal{D}$ qui a un comportement asymptotique significatif (voir [DFV] pour plus de détails). On ne s'intéresse qu'aux points $z_{0}$ dont le $k$-ème itéré $z_{k}$ appartient encore au disque $\mathcal{D}$ et on cherche à évaluer la répartition de ces itérés dans le disque $\mathcal{D}$. Tout ceci revient à étudier la densité conditionnelle $F_{k}:=\widetilde{F}_{k} / \varrho_{k}$ que nous nommerons tout simplement densité d'ordre $k$.

L'évènement $[L \geq k+1]$ est formé des éléments $z_{0}$ de $\mathcal{D}$ pour lesquels les $k$ premiers itérés $z_{j}=U^{j}\left(z_{0}\right)(1 \leq j \leq k)$ appartiennent tous au disque $\mathcal{D}$. On a donc

$$
[L \geq k+1]=\left.U\right|_{\mathcal{D}} ^{-k}(\mathcal{D})=\bigcup_{|h|=k} h(\mathcal{D}),
$$

d'après la définition même des homographies de hauteur $k$. Par analogie avec la terminologie d'intervalles fondamentaux, les disques $h(\mathcal{D})$, construits sur 
les intervalles fondamentaux $h(\mathcal{I})$, s'appellent les disques fondamentaux. Ils sont disjoints. Voici une figure représentant les disques fondamentaux correspondant à des homographies de hauteur $k \leq 4$ (coloriés suivant la parité de $k)$.

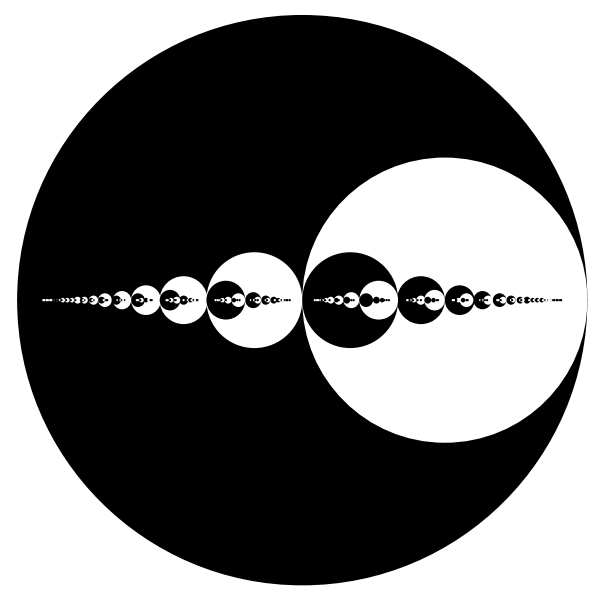

La distribution $\varrho_{k}$ et la densité $F_{k}$ dépendent toutes deux de la densité initiale $f$, et on les désignera respectivement par $\varrho_{k}[f]$ et $F_{k}[f]$, les notations $\varrho_{k}$ et $F_{k}$ étant réservées au cas où la densité initiale est uniforme. On remarque d'ailleurs, en observant la figure, que c'est le comportement de la densité initiale au voisinage de l'axe réel qui va avoir une influence importante sur l'asymptotique des grandeurs étudiées.

On va donc travailler avec des densités $f$ qui, considérées comme fonction de deux variables réelles $x$ et $y$, sont non nécessairement analytiques, et on introduit la notion de valuation au voisinage de l'axe réel.

DÉfinition. Une fonction $f$ définie sur $\mathcal{D} \backslash \mathcal{I}$ est dite de valuation $r$ $(r>-1)$ au voisinage de l'axe réel s'il existe une fonction $g$ définie sur $\mathcal{D}$, analytique en $x$ et $y$, continue sur $\overline{\mathcal{D}}$, positive sur $\mathcal{D}$ et strictement positive sur $\mathcal{I}$ pour laquelle

$$
f(x, y)=|y|^{r} g(x, y) \quad \text { pour tout point } z=x+i y \text { de } \mathcal{D} \backslash \mathcal{I} .
$$

La proposition suivante constitue le fondement du deuxième axe de ce travail : Elle montre que, pour une densité initiale quelconque, les deux objets étudiés (densité dynamique et distribution du nombre d'itérations) s'expriment en fonction des itérés des opérateurs de Ruelle-Mayer généralisés, ou non, pour des paramètres pris en $s=4$ ou en $(s, t)=(2,2)$ ou encore en $(s, t)=(2+r, 2+r)$ pour une densité de valuation $r$. 
Proposition 2. La distribution de probabilités $\varrho_{k}$ et la densité $F_{k}$ d'ordre $k$ s'expriment en fonction des itérés d'ordre $k$ des opérateurs de Ruelle-Mayer.

(i) Pour une densité uniforme, en fonction de l'opérateur usuel $\mathcal{G}_{4}$ [DFV],

$$
F_{k}(x, y)=\frac{4}{\pi \varrho_{k}} \mathcal{G}_{4}^{k}\left[\nu_{z, \bar{z}}^{(2,2)}\right](0) \text { avec } \varrho_{k}=\mathcal{G}_{4}^{k}\left[\frac{1}{(1+u)^{2}}\right](0),
$$

et

$$
\nu_{z, \bar{z}}^{(2,2)}(t)=\frac{1}{(1+z t)^{2}(1+\bar{z} t)^{2}} \quad(i c i, z=x+i y, \bar{z}=x-i y) .
$$

(ii) Pour une densité intégrable $f$ sur $\mathcal{D}$, en fonction de l'opérateur généralisé $\mathbf{G}_{2,2}$,

avec

$$
F_{k}[f](x, y)=\frac{4}{\pi \varrho_{k}[f]} \mathbf{G}_{2,2}^{k}[F](x+i y, x-i y)
$$

$$
\varrho_{k}[f]=\int_{\mathcal{D}} \mathbf{G}_{2,2}^{k}[F](x+i y, x-i y) d x d y,
$$

où la fonction $F$ de deux variables complexes est définie à partir de la fonction $f$ de deux variables réelles par la relation $F(x+i y, x-i y)=f(x, y)$.

(iii) Pour une densité $f$ de valuation $r$ de la forme $f(x, y)=|y|^{r} g(x, y)$, en fonction de l'opérateur généralisé $\mathbf{G}_{2+r, 2+r}$,

avec

$$
F_{k}[f](x, y)=\frac{4}{\pi \varrho_{k}[f]}|y|^{r} \mathbf{G}_{2+r, 2+r}^{k}[G](x+i y, x-i y)
$$

$$
\varrho_{k}[f]=\int_{\mathcal{D}}|y|^{r} \mathbf{G}_{2+r, 2+r}^{k}[G](x+i y, x-i y) d x d y,
$$

où la fonction $G$ de deux variables complexes est définie à partir de la fonction $g$ de deux variables réelles par la relation $G(x+i y, x-i y)=g(x, y)$.

Preuve. La preuve de (i) se trouve dans [DFV]. Dans le cas d'une mesure $\mu$ associée à une densité $f(x, y)$, en vertu de (17), la probabilité $\varrho_{k}[f]$ est donc égale à

$$
\operatorname{Pr}[L \geq k+1]=\sum_{|h|=k} \mu[h(\mathcal{D})]=\sum_{|h|=k} \int_{h(\mathcal{D})} f(x, y) d x d y .
$$

On effectue le changement de variable $z=h(Z)$ dans chaque disque $h(\mathcal{D})$, ou plus exactement le changement de variable $(x, y)=\widehat{h}(X, Y)$ associé à l'interprétation $\widehat{h}: \mathbb{R}^{2} \rightarrow \mathbb{R}^{2}$ de l'homographie $h: \mathbb{C} \rightarrow \mathbb{C}$. Il intervient alors le jacobien de la transformation $\widehat{h}$, égal à $\left|h^{\prime}(Z)\right|^{2}=|\widetilde{h}(Z)|^{4}$, avec la fonction $\widetilde{h}$ définie en (9), et, après interversion de la somme et de l'intégrale, 
on obtient

$$
\varrho_{k}[f]=\int_{\mathcal{D}} \sum_{|h|=k}|\widetilde{h}(x+i y)|^{4} f \circ \widehat{h}(x, y) d x d y .
$$

La fonction à intégrer s'exprime en fonction du $k$-ème itéré de l'opérateur $\mathcal{H}_{s}$ défini par la relation

$$
\mathcal{H}_{s}[f](x, y)=\sum_{|h|=1}|\widetilde{h}(x+i y)|^{s} f \circ \widehat{h}(x, y),
$$

qui donne par itération

$$
\mathcal{H}_{s}^{k}[f](x, y)=\sum_{|h|=k} \mid \widetilde{h}\left(x+\left.i y\right|^{s} f \circ \widehat{h}(x, y),\right.
$$

et on obtient une expression de $\varrho_{k}[f]$ faisant intervenir le $k$-ème itéré de l'opérateur $\mathcal{H}_{4}$,

$$
\varrho_{k}[f]=\int_{\mathcal{D}} \mathcal{H}_{4}^{k}[f](x, y) d x d y .
$$

Dans le cas où la densité est de valuation $r$, définie en (18), de la forme $f(x, y)=|y|^{r} g(x, y)$, on remarque la relation

$$
\mathcal{H}_{s}^{k}[f](x, y)=|y|^{r} \mathcal{H}_{s+2 r}^{k}[g](x, y) \quad \text { pour tout } k \geq 0,
$$

ce qui permet d'exprimer dans ce cas $\varrho_{k}[f]$ en fonction du $k$-ème itéré de l'opérateur $\mathcal{H}_{4+2 r}$. Le module intervenant dans la définition de $\mathcal{H}_{s}$ semble poser un problème, car les espaces sur lesquels on peut travailler commodément sont des espaces de fonctions holomorphes. On résout cette difficulté en travaillant dans $\mathbb{C} \times \mathbb{C}$ avec les opérateurs généralisés : On utilise la fonction $F$ définie par $f(x, y)=F(x+i y, x-i y)$ et la relation $|\widetilde{h}(x+i y)|^{2 s}$ $=\widetilde{h}(x+i y)^{s} \widetilde{h}(x-i y)^{s}$. On remarque de plus l'égalité $f \circ \widehat{h}(x, y)=$ $F(h(x+i y), h(x-i y))$ et finalement on obtient une relation entre l'opérateur $\mathcal{H}_{2 s}$ et l'opérateur généralisé $\mathbf{G}_{s, s}$ :

$$
\mathcal{H}_{2 s}^{k}[f](x, y)=\mathbf{G}_{s, s}^{k}[F](x+i y, x-i y) .
$$

La même démonstration prouve aussi que la densité $F_{k}[f](x, y)$ d'ordre $k$ est proportionnelle à

$$
\begin{aligned}
\mathcal{H}_{4}^{k}[f](x, y) & =\mathbf{G}_{2,2}^{k}[F](x+i y, x-i y) \\
& =\sum_{|h|=k} \widetilde{h}(x+i y)^{2} \widetilde{h}(x-i y)^{2} F(h(x+i y), h(x-i y)),
\end{aligned}
$$

dans le cas d'une densité quelconque et à

$$
\mathcal{H}_{4+2 r}^{k}[f](x, y)=\mathbf{G}_{2+r, 2+r}^{k}[G](x+i y, x-i y)
$$


dans le cas d'une densité de valuation $r$, le coefficient de proportionnalité assurant que

$$
\int_{\mathcal{D}} F_{k}[f](x, y) d x d y=1
$$

et constituant aussi le facteur de "conditionnement" de la densité.

Ainsi, les Propositions 1 et 2 montrent que les objets étudiés ici s'expriment en fonction des itérés des opérateurs de Ruelle-Mayer, généralisés ou non. C'est essentiellement une étude asymptotique que nous menons, et elle va donc dépendre des propriétés spectrales de ces opérateurs : les propriétés spectrales dominantes permettent alors de déterminer le terme principal, tandis que la vitesse de convergence ou le terme d'erreur vont dépendre des propriétés spectrales sous-dominantes.

On étudie donc ces opérateurs $\mathbf{G}_{s, t}$ qui se présentent comme des extensions formelles et naturelles des opérateurs $\mathcal{G}_{s+t}$. L'idée n'est pas d'en faire une étude directe, mais de décrire comment les propriétés essentielles des opérateurs à une variable se transmettent aux opérateurs à deux variables. C'est pourquoi on décrit, dans la section suivante, les principales propriétés des opérateurs de Ruelle-Mayer à une variable.

2. Propriétés des opérateurs de Ruelle-Mayer à une variable. Après avoir rappelé les notions de nucléarité et de $u_{0}$-positivité, on décrit les propriétés des opérateurs classiques afin de montrer par la suite l'héritage de ces propriétés par les opérateurs généralisés.

2.1. Rappels sur les opérateurs nucléaires d'ordre 0. Soit $B$ un espace de Banach et $B^{\star}$ son espace dual. Un opérateur $\mathcal{L}: B \rightarrow B$ est nucléaire d'ordre 0 s'il admet la représentation

$$
\mathcal{L}=\sum_{i \in I} \mu_{i} e_{i}^{\star} \otimes e_{i} \text { ou encore } \mathcal{L}[f]=\sum_{i \in I} \mu_{i} e_{i}^{\star}(f) e_{i} \text { pour tout } f \in B,
$$

avec $e_{i} \in B, e_{i}^{\star} \in B^{\star}$ vérifiant $\left\|e_{i}\right\|=\left\|e_{i}^{\star}\right\|=1$ et $\mu_{i} p$-sommable pour tout $p>0$ (i.e. $\sum\left|\mu_{i}\right|^{p}<\infty$ ).

De tels opérateurs ont été introduits et étudiés par Grothendieck [Gr1], [Gr2]. Ils sont compacts. Mieux, on peut justifier sur cette classe la plupart des calculs matriciels classiques. On définit ainsi la trace d'un tel opérateur,

$$
\operatorname{Tr} \mathcal{L}=\sum_{i \in I} \mu_{i} e_{i}^{\star}\left(e_{i}\right) \text {, égale aussi à } \operatorname{Tr} \mathcal{L}=\sum_{i \in I} \lambda_{i},
$$

où les $\lambda_{i}$ sont les valeurs propres de $\mathcal{L}$ comptées avec leur multiplicité algébrique. Les traces des itérés de $\mathcal{L}$ sont également bien définis. Le déterminant de Fredholm de $\mathcal{L}$, qui représente l'analogue du polynôme caractéristique, 


$$
\operatorname{det}(I-z \mathcal{L}):=\prod_{i \in I}\left(1-\lambda_{i} z\right),
$$

où les $\lambda_{i}$ sont les valeurs propres de $\mathcal{L}$ comptées avec leur multiplicité algébrique, admet alors l'expression

$$
\operatorname{det}(I-z \mathcal{L})=\exp [-\operatorname{Tr} \log (I-z \mathcal{L})]=\exp \left[-\sum_{m=1}^{\infty} \frac{z^{m}}{m} \operatorname{Tr} \mathcal{L}^{m}\right] .
$$

Ainsi, les opérateurs nucléaires d'ordre 0 ont toutes les bonnes propriétés qui justifient un calcul matriciel sur des matrices infinies. Certains espaces de Banach sont spécialement intéressants car tout opérateur borné y est nucléaire d'ordre 0. C'est le cas [Gr1], [Gr2] des espaces de fonctions holomorphes $A_{\infty}(\mathcal{V})$ ou $B_{\infty}(\mathcal{V})$ qui seront largement utilisés dans la suite.

2.2. Rappels sur les opérateurs $u_{0}$-positifs. Ces opérateurs ont été introduits par Krasnosel'skiǔ [Kr]; ils généralisent les opérateurs positifs de la dimension finie et possèdent des propriétés spectrales dominantes.

Un ensemble $K$ d'un espace de Banach réel $B$ est appelé un cône propre si

(i) pour tout réel $\varrho>0$ et tout $f$ de $K$, $\varrho f \in K$,

(ii) $K \cap-K=\{0\}$.

Un cône propre est appelé reproductif si $B=K-K$, i.e. tout élément $f$ de $B$ s'écrit comme la différence de deux éléments de $K$.

Soit $K$ un cône propre, reproductif et d'intérieur $K$ Kon vide. On dit que $\mathcal{L}: B \rightarrow B$ est positif (par rapport au cône $K$ ) si $\mathcal{L}(K)$ est inclus dans $K$. Soit $u_{0}$ un élément de $\stackrel{\circ}{K}$; on dit que l'opérateur positif $\mathcal{L}$ est $u_{0}$-positif par rapport au cône $K$ si pour tout élément $f$ non nul de $K$, il existe un entier $p$ et deux réels $\alpha$ et $\beta$ strictement positifs pour lesquels

$$
\beta u_{0} \leq \mathcal{L}^{p}[f] \leq \alpha u_{0},
$$

où l'ordre est défini en relation avec $K: f \leq g$ si et seulement si $g-f \in K$.

Alors, d'après un théorème dû à Krasnosel'skiı̌ $[\mathrm{Kr}]$, un opérateur $\mathcal{L}$ compact et $u_{0}$-positif satisfait à une propriété de type Perron-Frobenius : il a un unique vecteur propre $g$ dans $\stackrel{\circ}{K}$ et la valeur propre associée $\lambda$ est simple, et strictement plus grande en valeur absolue que les autres valeurs propres.

Nous décrivons maintenant les principales propriétés dont jouissent les opérateurs de Ruelle-Mayer à une variable : Sur un espace de Banach adapté, ils sont nucléaires d'ordre 0, et leur spectre est donc discret. Sur un espace de Hilbert convenable, ils sont isomorphes à des opérateurs intégraux, et sont donc diagonalisables. De plus, ces deux espaces de définition, espace de Banach et espace de Hilbert, sont très fortement liés. Enfin, si le paramètre $s$ est réel, ces opérateurs possèdent un spectre réel et satisfont à une propriété 
de positivité de type Perron-Frobenius, qui prouve l'existence d'objets spectraux dominants, qui se prolongent lorsque le paramètre $s$ reste au voisinage de l'axe réel.

2.3. Propriétés des opérateurs de Ruelle-Mayer usuels : nucléarité. On désigne par $\mathcal{P}_{\varrho}$ le demi-plan $\{z \mid \Re(z)>\varrho\}$, et on pose $\mathcal{P}:=\mathcal{P}_{-1 / 2}$. On considère un intervalle ouvert borné $\mathcal{J}$ qui contient strictement le segment $[0,1]$, et on désigne par $\mathcal{V}$ le disque ouvert de diamètre $\mathcal{J}$. On exige de plus que $\mathcal{V}$ soit inclus dans $\mathcal{P}$ et que le demi-plan $\overline{\mathcal{P}}$ soit envoyé dans le disque $\mathcal{V}$ par toutes les homographies de hauteur 1, i.e.

$$
h(\overline{\mathcal{P}}) \subset \mathcal{V} \quad \text { pour }|h|=1 .
$$

Ceci entraîne en particulier que le disque $\mathcal{V}$ est strictement conservé par toutes les homographies de hauteur 1 ,

$$
h(\overline{\mathcal{V}}) \subset \mathcal{V} \quad \text { pour }|h|=1 .
$$

Une configuration-type est fondée sur un intervalle $\mathcal{J}_{\delta}$ de la forme $]-\delta, 2+\delta[$ $(0<\delta \leq 1 / 2)$, et Mayer ou Hensley choisissent $\delta=1 / 2$. Ici, nous travaillons avec un paramètre $\delta$ vérifiant $0<\delta \leq 1 / 4$ et nous utilisons communément $\delta=1 / 4$ : Dans la suite, $\mathcal{J}$ et $\mathcal{V}$ désignent respectivement le segment et le disque ouvert de centre 1 et de rayon $5 / 4$. Cependant, tous les résultats de la section s'adaptent à un rayon de la forme $1+\delta$ avec $0<\delta \leq 1 / 4$.

On définit les opérateurs $\mathcal{G}_{s}$, pour un paramètre $s$ vérifiant $\Re(s)>1$, sur l'ensemble $A_{\infty}(\mathcal{V})$ formé par les fonctions $f$ holomorphes dans $\mathcal{V}$ et continues $\operatorname{sur} \overline{\mathcal{V}}$. Muni de la norme sup $\|\cdot\|$ définie par

$$
\|f\|=\sup \{|f(z)| \mid z \in \mathcal{V}\}
$$

cet ensemble est un espace de Banach.

Chaque opérateur $\mathcal{G}_{s}$ s'exprime, ainsi que ses itérés, comme la somme d'opérateurs $\mathcal{C}_{s}^{(h)}$ définis en (10). Chacun de ces opérateurs est borné sur $A_{\infty}(\mathcal{V})$ et y est donc nucléaire d'ordre 0 . D'après des résultats classiques repris dans ce contexte par Mayer [Ma1], son spectre, formé de valeurs propres simples, est égal à la progression géométrique de premier terme $\mu_{0}=$ $\widetilde{h}\left(z^{\star}\right)^{s}$ et de raison $\mu=(-1)^{k} \widetilde{h}\left(z^{\star}\right)^{2}$, où $k$ est la hauteur de l'homographie et $z^{\star}$ est l'unique point fixe de $h$ situé dans $\mathcal{V}$. On a donc

$$
\operatorname{Sp} \mathcal{C}_{s}^{(h)}=\left\{\mu_{n}=\mu_{0} \mu^{n}=\widetilde{h}\left(z^{\star}\right)^{s}(-1)^{k n} \widetilde{h}\left(z^{\star}\right)^{2 n} \mid n \in \mathbb{N}\right\} .
$$

De plus, le vecteur propre correspondant à $\mu_{n}$ vérifie les conditions

$$
f^{(i)}\left(z^{\star}\right)=0 \text { pour } i<n \text { et } f^{(n)}\left(z^{\star}\right) \neq 0 .
$$

Puisque $\mathcal{G}_{s}$ est lui-même borné, comme série convergente d'opérateurs bornés, il est également nucléaire d'ordre 0 et ce qui précède permet de donner une expression de sa trace et de celle de ses itérés, et, via le determinant de Fredholm, d'avoir accès au spectre de l'opérateur $\mathcal{G}_{s}$. 
2.4. Représentation intégrale des opérateurs de Ruelle-Mayer usuels. Les opérateurs de Ruelle-Mayer jouissent de propriétés plus fortes quand ils opèrent sur des espaces de fonctions plus restreints, qui sont des espaces de Hilbert appelés les espaces de Hardy. Cette structure permet de montrer que ces opérateurs sont diagonalisables, et que leur spectre est réel dès que le paramètre $s$ l'est. On peut alors évaluer précisément la norme de ces opérateurs. Comme, de plus, ces espaces de Hilbert sont fortement comparables aux espaces de Banach initiaux, on peut "transporter" la plupart des résultats obtenus dans les espaces de Banach initiaux.

Ce point de vue a été adopté d'abord par Babenko [Ba], puis généralisé dans [Ma1], [Ma4]. On considère les demi-plans $\mathcal{P}_{\varrho}=\{z \mid \Re(z)>\varrho\}$, $\mathcal{P}=\{z \mid \Re(z)>-1 / 2\}$ et l'espace de Hilbert $\mathbf{H}_{s}$ formé des fonctions $f$ holomorphes sur $\mathcal{P}$, bornées sur tout $\mathcal{P}_{\varrho}(\varrho>-1 / 2)$ et admettant une représentation intégrale de la forme

$$
f(z)=\int_{0}^{\infty} e^{-w z} \phi(w) w^{(s-1) / 2} \frac{d w}{e^{w}-1},
$$

où $\phi$ est de carré intégrable par rapport à la mesure $d m(w)$ de densité $1 /\left(e^{w}-1\right)$. La norme associée $|\cdot|\langle s\rangle$ est définie par

$$
|f|_{\langle s\rangle}^{2}=\int_{0}^{\infty}|\phi(w)|^{2} \frac{d w}{e^{w}-1} .
$$

ThÉORÈme 1 [Babenko, Mayer]. Soit s un paramètre complexe vérifiant $\Re(s)>1$.

(i) L'opérateur $\mathcal{G}_{s}: \mathbf{H}_{s} \rightarrow \mathbf{H}_{s}$ est isomorphe à un opérateur intégral; c'est un opérateur normal, qui est donc diagonalisable sur l'espace $\mathbf{H}_{s}$ dans une base orthonormale; de plus, pour $s$ réel, $s>1$, l'opérateur $\mathcal{G}_{s}$ est autoadjoint et son spectre est réel.

(ii) Les deux normes $\|\cdot\|$ et $|\cdot|\langle s\rangle$ sont quasi-équivalentes dans le sens suivant : La fonction $\mathcal{G}_{s}[f]$ est élément de $\mathbf{H}_{s}$ dès que la fonction $f$ est élément de $A_{\infty}(\mathcal{V})$ et il existe deux constantes $A(s)$ et $B(s)$ pour lesquelles on a la double inégalité

$$
\begin{aligned}
\|f\| \leq A(s)|f|_{\langle s\rangle} & \text { pour } f \in \mathbf{H}_{s}, \\
\left|\mathcal{G}_{s}[f]\right|_{\langle s\rangle} \leq B(s)\|f\| & \text { pour } f \in A_{\infty}(\mathcal{V}) .
\end{aligned}
$$

(iii) Il y a coïncidence entre les deux spectres, le spectre de $\mathcal{G}_{s}$ dans le Banach $A_{\infty}(\mathcal{V})$ et le spectre de $\mathcal{G}_{s}$ dans le Hilbert $\mathbf{H}_{s}$.

Pr e u ve. Nous donnons les grandes lignes; les preuves détaillées se trouvent dans [Ma1]. 
(i) Sur l'espace $\mathbf{H}_{s}$, l'opérateur $\mathcal{G}_{s}$ s'exprime sous la forme

$$
\mathcal{G}_{s}[f](z)=\int_{0}^{\infty} e^{-w z} \mathcal{K}_{s}[\phi](w) w^{(s-1) / 2} \frac{d w}{e^{w}-1},
$$

où $\mathcal{K}_{s}$ est un opérateur intégral faisant intervenir la fonction de Bessel $J_{s-1}$ d'indice $s-1$,

$$
J_{s-1}(u)=\sum_{k=0}^{\infty}\left(\frac{u}{2}\right)^{2 k+s-1} \frac{(-1)^{k}}{k ! \Gamma(k+s)}
$$

sous la forme

$$
\mathcal{K}_{s}[\phi](w)=\int_{0}^{\infty} J_{s-1}(2 \sqrt{v w}) \phi(v) \frac{d v}{e^{v}-1} .
$$

Ainsi, dans cet espace $\mathbf{H}_{s}, \mathcal{G}_{s}$ est isomorphe à un opérateur intégral dont le noyau est la fonction de Bessel d'indice $s-1$. Ceci prouve (i).

(ii) On prouve (28) avec l'inégalité de Cauchy-Schwarz : pour tout $s$ vérifiant $\Re(s)>1$ et toute fonction $f \in \mathbf{H}_{s}$, il existe une constante $A(s)$ pour laquelle

$$
|f(z)| \leq A(s)|f|_{\langle s\rangle} \quad \text { pour tout } z \in \mathcal{V} .
$$

Pour $\Re(s) \geq 2$, l'espace $\mathbf{H}_{s}$ est identique [Ma1] à l'espace $\widehat{\mathbf{H}}_{s}$ formé des fonctions holomorphes sur $\mathcal{P}$, bornées sur tout $\mathcal{P}_{\varrho}(\varrho>-1 / 2)$, et telles que

$$
\int_{\mathcal{B}}|x|^{\sigma-2}|f(z)|^{2} d x d y<\infty .
$$

Ici, on pose $\sigma=\Re(s), z=x+i y$, et $\mathcal{B}$ désigne la bande $\mathcal{B}=\{z \mid-1 / 2<$ $\Re(z) \leq 0\}$. En utilisant le théorème de Plancherel, on exprime la norme $|f|_{\langle s\rangle}$ en fonction de l'intégrale ci-dessus, sous la forme

$$
|f|_{\langle s\rangle}^{2}=\frac{2^{\sigma-2}}{\pi \Gamma(\sigma-2)} \int_{\mathcal{B}}|x|^{\sigma-2}|f(z)|^{2} d x d y \quad(\sigma=\Re(s), z=x+i y) .
$$

Ceci permet de prouver que, pour $\Re(s) \geq 2$, la fonction $\mathcal{G}_{s}[f]$ est élément de $\mathbf{H}_{s}$ dès que la fonction $f$ est élément de $A_{\infty}(\mathcal{V})$. On utilise pour cela deux inégalités : la première se déduit de $(30)$ pour $\Re(s) \geq 2$ :

$$
\left|\mathcal{G}_{s}[f]\right|_{\langle s\rangle} \leq\|f\| \sum_{m \geq 1}\left|\frac{1}{(m+z)^{s}}\right|_{\langle s\rangle},
$$

et la seconde s'obtient en calculant directement l'élément $\phi$ associé à chaque fonction de $\mathbf{H}_{s_{0}}$ de la forme $z \rightarrow 1 /(m+z)^{s}$ lorsque $\sigma:=\Re(s)$ et $\sigma_{0}:=\Re\left(s_{0}\right)$ vérifient $2 \sigma-\sigma_{0}>1$. On a alors

$$
\sum_{m \geq 1}\left|\frac{1}{(m+u)^{s}}\right|_{\left\langle s_{0}\right\rangle} \leq \frac{1}{|\Gamma(s)|} D\left(2 \sigma-\sigma_{0}\right)
$$


avec

$$
D(\alpha)=[\alpha \Gamma(\alpha)]^{1 / 2} \widetilde{\zeta}\left(\frac{\alpha+1}{2}\right),
$$

où $\widetilde{\zeta}$ représente la somme des termes d'indice impair de la fonction $\zeta$. Cette inégalité s'applique en particulier pour $s=s_{0}$, et $\sigma:=\Re(s)>1$, et en remplaçant alors (32) dans (31), on obtient (29) dans le cas $\Re(s) \geq 2$.

Lorsque $s$ vérifie $1<\Re(s)<2$, l'espace $\mathbf{H}_{s}$ est identique à l'espace formé par les fonctions $f$ holomorphes sur $\mathcal{P}$, bornées sur tout $\mathcal{P}_{\varrho}(\varrho>-1 / 2)$, s'annulant pour $\Re(z) \rightarrow \infty$, dont la dérivée $f^{\prime}$ appartient à l'espace $\widehat{\mathbf{H}}_{s+2}$. En utilisant la relation

$$
\frac{d}{d z} \mathcal{G}_{s}[f](z)=-s \mathcal{G}_{s+1}[f](z)-\mathcal{G}_{s+2}\left[f^{\prime}\right](z),
$$

et les relations (31), (32), appliquées aux fonctions du second membre, on démontre alors que, pour $s$ vérifiant $1<\Re(s)<2$, la fonction $\mathcal{G}_{s}[f]$ est également élément de $\mathbf{H}_{s}$ dès que la fonction $f$ est élément de $A_{\infty}(\mathcal{V})$. On en déduit finalement (ii), puis (iii).

2.5. Propriétés spectrales dominantes des opérateurs de Ruelle-Mayer usuels pour une valeur réelle du paramètre $s$. Si $s$ est réel, l'opérateur $\mathcal{G}_{s}$ est $u_{0}$-positif et satisfait donc une propriété de Perron-Frobenius, qui montre l'existence de propriétés spectrales dominantes.

ThÉonÈme 2 [Mayer]. (i) Pour un réel $s>1$, l'opérateur $\mathcal{G}_{s}: A_{\infty}(\mathcal{V}) \rightarrow$ $A_{\infty}(\mathcal{V})$ a une valeur propre dominante $\lambda(s)$ qui est simple, positive, et strictement plus grande que toutes les autres valeurs propres en valeur absolue. Le vecteur propre correspondant $f_{s}$ est strictement positif sur $\mathcal{J}$. $L$ 'opérateur adjoint $\mathcal{G}_{s}^{\star}: A_{\infty}^{\star}(\mathcal{V}) \rightarrow A_{\infty}^{\star}(\mathcal{V})$ a un vecteur propre dominant $f_{s}^{\star}$ correspondant à $\lambda(s)$ qui vérifie $f_{s}^{\star}[f]>0$ pour $f>0$ sur $\mathcal{J}$. Si $\mathcal{P}_{s}$ désigne la projection sur le sous-espace dominant définie par $\mathcal{P}_{s}=f_{s}^{\star} \otimes f_{s}$, alors $\mathcal{G}_{s}$ admet la représentation

$$
\mathcal{G}_{s}=\lambda(s) \mathcal{P}_{s}+\mathcal{N}_{s},
$$

où $\mathcal{P}_{s} \circ \mathcal{N}_{s}=\mathcal{N}_{s} \circ \mathcal{P}_{s}=0$. Si $f$ est une fonction de $A_{\infty}(\mathcal{V})$, on a

$$
\mathcal{G}_{s}^{k}[f](z)=\lambda(s)^{k} f_{s}^{\star}[f] f_{s}(z)+\mathcal{N}_{s}^{k}[f](z)
$$

pour tout $k \geq 1$ et tout $z$ dans $\mathcal{V}$.

(ii) Le rapport spectral $\varrho(s)$, égal au rapport entre le rayon spectral $\mu(s)$ de $\mathcal{N}_{s}$ et celui de $\mathcal{G}_{s}$, est strictement plus petit que 1 .

(iii) Pour une fonction $f$ de $\mathbf{H}_{s}$ et pour tout $k \geq 0$, on a

$$
\left|\frac{\mathcal{G}_{s}^{k}[f]}{\lambda(s)^{k}}-f_{s}^{\star}[f] f_{s}\right|_{\langle s\rangle} \leq|f|_{\langle s\rangle} \varrho(s)^{k} .
$$


(iv) Pour toute fonction $f$ de $A_{\infty}(\mathcal{V})$ strictement positive sur $\mathcal{J}$, on a

$$
\frac{\mathcal{G}_{s}^{k}[f](z)}{\lambda(s)^{k}}=f_{s}^{\star}[f] f_{s}(z)\left(1+O\left(\varrho(s)^{k}\right)\right)
$$

pour tout entier $k$. La constante du $O$ est uniforme sur $\mathcal{V}$ et dépend de $f$ et de $s$.

Preuve. (i) et (ii) : Si $s$ est réel $(s>1)$, l'opérateur $\mathcal{G}_{s}$ a un spectre réel et laisse stable l'espace $A_{\infty}^{\mathbb{R}}(\mathcal{V})$ formé par les éléments de $A_{\infty}(\mathcal{V})$ qui sont réels sur $\mathcal{J}$; c'est un espace de Banach réel, et l'ensemble $K$ des fonctions de $A_{\infty}(\mathcal{V})$ dont la restriction à $\mathcal{J}$ est positive ou nulle y forme un cône propre, reproductif et d'intérieur $K$ non vide. La fonction constante $u_{0}=1$ est un élément de $\stackrel{\circ}{K}$ et l'opérateur $\mathcal{G}_{s}$, ou plutôt sa restriction à $A_{\infty}^{\mathbb{R}}(\mathcal{V})$, est $u_{0}$-positif par rapport au cône $K$ [Ma1].

Alors on peut appliquer le théorème de Krasnosel'skiǔ, et comme le spectre de $\mathcal{G}_{s}$ est réel, les spectres de $\mathcal{G}_{s}$ et de sa restriction à $A_{\infty}^{\mathbb{R}}(\mathcal{V})$ coïncident.

(iii) Puisque l'opérateur $\mathcal{N}_{s}$ est auto-adjoint dans $\mathbf{H}_{s}$, sa norme $\left|\mathcal{N}_{s}\right|_{\langle s\rangle}$ dans $\mathbf{H}_{s}$ est égale au rayon spectral $\mu(s)$.

(iv) Remarquons d'abord que si on travaille uniquement dans l'espace de Banach, on peut obtenir une version plus faible de (iv), où la quantité $O\left(\varrho(s)^{k}\right)$ est remplacée par un terme de la forme $O\left(\alpha^{k}\right)$ où l'on peut choisir pour $\alpha$ tout nombre inférieur à 1 et majorant strictement le rapport spectral $\varrho(s)$. En effet, le théorème du rayon spectral compare de manière asymptotique la norme $\left\|\mathcal{N}_{s}\right\|$ de l'opérateur $\mathcal{N}_{s}$ dans l'espace de Banach avec le rayon spectral $\mu(s)$ de cet opérateur puisqu'il montre l'égalité

$$
\mu(s)=\lim _{k \rightarrow \infty}\left\|\mathcal{N}_{s}^{k}\right\|^{1 / k} .
$$

Pour tout $\alpha>\varrho(s)$, il existe donc une constante $A$ (dépendant de $\alpha$ ) pour laquelle, pour tout $k \geq 1$,

$$
\frac{1}{\lambda(s)^{k}}\left\|\mathcal{N}_{s}^{k}\right\| \leq A \alpha^{k}
$$

ce qui ne permet pas d'atteindre la véritable vitesse de convergence.

En utilisant les espaces de Hilbert $\mathbf{H}_{s}$ et en les "comparant" avec les espaces de Banach $A_{\infty}(\mathcal{V})$ grâce au Théorème 1 , on obtient le résultat plus fort annoncé : Pour tout $f$ dans l'espace de Banach $A_{\infty}(\mathcal{V}), \mathcal{G}_{s}[f]$ est dans l'espace de Hilbert $\mathbf{H}_{s}$, et donc $\mathcal{N}_{s}^{k}[f]=\mathcal{N}_{s}^{k-1}\left[\mathcal{G}_{s}[f]\right]$ a une norme dans l'espace de Hilbert qui vérifie, grâce à (iii),

$$
\left|\mathcal{N}_{s}^{k-1}\left[\mathcal{G}_{s}[f]\right]\right|_{\langle s\rangle} \leq \mu(s)^{k-1}\left|\mathcal{G}_{s}[f]\right|_{\langle s\rangle} .
$$

Comme les normes $\|\cdot\|$ et $|\cdot|_{\langle s\rangle}$ sont presque équivalentes, en vertu de (28) 
et (29), on déduit donc, pour $k \geq 1$, la majoration

$$
\frac{1}{\lambda(s)^{k}}\left\|\mathcal{N}_{s}^{k}[f]\right\| \leq C \varrho(s)^{k}\|f\|,
$$

où $C$ est une constante qui dépend de $s$ mais non de $f$.

Pour un réel $s>1$, la valeur propre dominante $\lambda(s)$ peut alors être définie par

$$
\lambda(s)=\lim _{k}\left(\sum_{Q_{k}} \frac{1}{Q_{k}^{s}}\right)^{1 / k} .
$$

Il suffit d'appliquer ce qui précède à la fonction 1 qui est un élément de $A_{\infty}(\mathcal{V})$. Le plus petit des continuants $Q_{k}$ est obtenu, grâce à (3), quand tous les $m_{i}$ sont égaux à 1 ; c'est donc le $k$-ème nombre de Fibonacci $\phi_{k}$ qui vérifie $\lim _{k} \phi_{k}^{1 / k}=\phi$ où $\phi$ est le nombre d'or égal à $(1+\sqrt{5}) / 2$, ce qui montre que $s \rightarrow \lambda(s)$ vérifie, pour tout $u \geq 0$,

$$
\lambda(s+u) \leq \frac{1}{\phi^{u}} \lambda(s),
$$

et définit donc une fonction strictement décroissante de $s$.

2.6. Propriétés spectrales dominantes au voisinage de $s=2$. La Proposition 1 fait intervenir les opérateurs $\mathcal{G}_{s}$ lorsque $s$ est au voisinage de 2 . Il faut donc utiliser les propriétés spectrales dominantes de $\mathcal{G}_{s}$ qui vont s'obtenir par perturbation de celles de l'opérateur $\mathcal{G}_{2}$.

Pour $s=2$, l'opérateur $\mathcal{G}_{s}$ a des propriétés spectrales dominantes bien connues; la valeur propre dominante $\lambda(2)$ est égale à 1 , le rayon spectral de $\mathcal{N}_{2}$, déterminé d'abord par Wirsing [Wi], vaut

$$
\mu(2) \approx 0.303663 \text {, }
$$

et est appelée communément la constante de Gauss-Kuz'min-Wirsing. Le vecteur propre dominant $f_{2}$, correspondant à la densité-limite de l'algorithme des fractions continues, et le vecteur propre dominant $f_{2}^{\star}$ de l'opérateur adjoint sont tous deux explicites et égaux respectivement à

$$
f_{2}(z)=\frac{1}{\log 2} \cdot \frac{1}{1+z} \text { et } f_{2}^{\star}[f]=\int_{0}^{1} f(x) d x .
$$

Sur un voisinage de $s=2$, l'opérateur $\mathcal{G}_{s}$ définit une application analytique $s \rightarrow \mathcal{G}_{s}$. En utilisant la théorie des perturbations sur l'espace $A_{\infty}(\mathcal{V})$, Faivre $[\mathrm{Fa}]$ montre que les propriétés spectrales dominantes de $\mathcal{G}_{2}$ se prolongent sur un voisinage complexe de $s=2$.

ThÉORÈME 3. Soit $\alpha$ un nombre réel strictement supérieur à la constante $\mu(2)$ dite de Gauss-Kuz'min-Wirsing. Il existe un voisinage complexe $\Omega$ de 2 pour lequel les propriétés spectrales dominantes de $\mathcal{G}_{2}: A_{\infty}(\mathcal{V}) \rightarrow A_{\infty}(\mathcal{V})$ 
se prolongent à $\mathcal{G}_{s}$ : les quatre quantités $\lambda(s), f_{s}, f_{s}^{\star}\left(\right.$ et donc $\left.\mathcal{P}_{s}\right), \mathcal{N}_{s}$ y sont bien définies, y représentent les objets spectraux dominants de $\mathcal{G}_{s}$ et sont analytiques en $s$. De plus, sur le même voisinage $\Omega$, et pour toute fonction $f$ de $A_{\infty}(\mathcal{V})$ strictement positive sur $\mathcal{J}$, on a

$$
\mathcal{G}_{s}^{k}[f](z)=\lambda(s)^{k} f_{s}(z) f_{s}^{\star}[f]\left(1+O\left(\alpha^{k}\right)\right)
$$

pour tout $k \geq 1$, et pour tout point $z$ de $\mathcal{V}$. La constante du $O$ est uniforme sur $\Omega \times \mathcal{V}$. Elle dépend de $f$ et de $\alpha$.

Preuve. C'est une application simple de la théorie des perturbations. Contrairement à la preuve précédente, on peut travailler seulement dans l'espace de Banach, puisque, de toute façon, l'argument de continuité obligera à choisir $\alpha$ strictement plus grand que le rayon spectral $\mathcal{N}_{2}$, qui est lui égal à la constante de Gauss-Kuz'min-Wirsing. La preuve est omise, elle sera faite dans un cadre plus général pour le Théorème 6 .

3. Propriétés des opérateurs de Ruelle-Mayer à deux variables. On montre ici que la famille des opérateurs $\mathbf{G}_{s, t}$ hérite des principales propriétés des opérateurs usuels, dues pour une part importante à leur nucléarité et à leur positivité : l'opérateur $\mathbf{G}_{s, t}$ a essentiellement les mêmes propriétés spectrales dominantes que $\mathcal{G}_{s+t}$. On peut donc étudier le comportement asymptotique des principaux objets liés à l'analyse des algorithmes de Gauss et d'Euclide. Par ailleurs, on peut définir une structure d'espace de Hilbert qui généralise celle qui existe pour les opérateurs usuels. Cette structure va permettre d'évaluer les termes-reste en fonction des propriétés spectrales subdominantes.

On définit $\mathbf{G}_{s, t}$, pour deux paramètres $s$ et $t$ vérifiant $\Re(s+t)>1$. Certaines des propriétés qui vont être énoncées sont valables pour tout couple de complexes $(s, t)$ vérifiant $\Re(s+t)>1$. Nous supposerons cependant dans toute la suite que le couple $(s, t)$ vérifie les conditions plus restrictives suivantes :

$$
\Re(s+t)>1, \quad \Re(s) \geq 1, \quad \Re(t)>-1 .
$$

On conserve par ailleurs les notations de la section 2 : On désigne par $\mathcal{P}_{\varrho}$ le demi-plan $\{z \mid \Re(z)>\varrho\}$, et on pose $\mathcal{P}:=\mathcal{P}_{-1 / 2}$. Les symboles $\mathcal{J}$ et $\mathcal{V}$ désignent respectivement le segment et le disque ouvert de centre 1 et de rayon $5 / 4$.

La famille $\mathbf{G}_{s, t}$ est définie sur l'ensemble $B_{\infty}(\mathcal{V})$ formé par les fonctions $F$ holomorphes dans $\mathcal{V} \times \mathcal{V}$ et continues sur $\overline{\mathcal{V}} \times \overline{\mathcal{V}}$. Muni de la norme sup $\|\cdot\|$ définie par

$$
\|F\|=\sup \{|F(u, v)| \mid(u, v) \in \mathcal{V} \times \mathcal{V}\},
$$

cet ensemble est un espace de Banach. 
Chaque opérateur $\mathbf{G}_{s, t}$ s'exprime, ainsi que ses itérés, comme une somme convergente d'opérateurs $\mathbf{C}_{s, t}^{(h)}$ définis en (10). Chacun d'entre eux est borné sur $B_{\infty}(\mathcal{V})$ et y est donc nucléaire d'ordre 0 , de sorte que l'opérateur $\mathbf{G}_{s, t}$ lui-même est nucléaire d'ordre 0 .

3.1. Les valeurs propres de $\mathbf{G}_{s, t}$. On relie ici, grâce au résultat suivant, le spectre de $\mathbf{G}_{s, t}$ à celui des opérateurs $\mathcal{G}_{s+t+2 l}$ :

ThÉORÈme 4. Les traces des opérateurs $\mathbf{G}_{s, t}: B_{\infty}(\mathcal{V}) \rightarrow B_{\infty}(\mathcal{V})$ et de leurs itérés s'expriment en fonction des traces des opérateurs classiques agissant sur $A_{\infty}(\mathcal{V})$ :

$$
\operatorname{Tr} \mathbf{G}_{s, t}^{k}=\sum_{l \geq 0}(-1)^{k l} \operatorname{Tr} \mathcal{G}_{s+t+2 l}^{k} \quad \text { pour tout } k \geq 0 .
$$

Il s'ensuit une égalité entre les déterminants de Fredholm

$$
\operatorname{det}\left(I-z \mathbf{G}_{s, t}\right)=\prod_{l \geq 0} \operatorname{det}\left(I-(-1)^{l} z \mathcal{G}_{s+t+2 l}\right)
$$

et l'identité des spectres

$$
\operatorname{Sp} \mathbf{G}_{s, t}=\bigcup_{l \geq 0}(-1)^{l} \operatorname{Sp} \mathcal{G}_{s+t+2 l},
$$

où la multiplicité algébrique d'une valeur propre $\lambda$ de $\mathbf{G}_{s, t}$ est égale à la somme des multiplicités algébriques de la valeur propre $(-1)^{l} \lambda$ pour chacun des opérateurs $\mathcal{G}_{s+t+2 l}$.

R e marque. Le spectre de $\mathbf{G}_{s, t}$ ne dépend donc que de la somme $s+t$ et il est réel dès que la somme $s+t$ l'est.

Preuve du Théorème 4. On adapte un théorème de Mayer [Ma2] afin de déterminer, pour toute homographie $h$, le spectre de $\mathbf{C}_{s, t}$. Le théorème de Mayer décrit le spectre d'un opérateur $\mathcal{R}$ de la forme

$$
\mathcal{R}[f](z)=\phi(z) f \circ \psi(z)
$$

défini sur l'espace des fonctions holomorphes $f$ sur $\Omega$ et continues sur $\bar{\Omega}$ pour un ouvert $\Omega$ de $\mathbb{C}^{m}$. La fonction $\psi$ est une fonction du même espace qui envoie strictement $\Omega$ sur lui-même. Elle a alors un seul point fixe $z^{\star}$ à l'intérieur de $\Omega$ en lequel les valeurs propres de $\psi^{\prime}\left(z^{\star}\right)$ sont strictement inférieures à 1 en valeur absolue. Le spectre de $\mathcal{R}$ est égal à l'ensemble

$$
\operatorname{Sp} \mathcal{R}=\bigcup_{l \geq 0} \phi\left(z^{\star}\right)\left[\operatorname{Sp} \psi^{\prime}\left(z^{\star}\right)\right]^{l},
$$

avec la convention suivante : Pour $A=\left\{a_{i} \mid 1 \leq i \leq m\right\}$, l'ensemble $A^{l}$ désigne l'ensemble des produits commutatifs ordonnés sous la forme

$$
A^{l}=\left\{a_{i_{1}} \ldots a_{i_{l}} \mid 1 \leq i_{1} \leq \ldots \leq i_{l} \leq m\right\} .
$$


En appliquant le théorème de Mayer, on déduit que, pour toute homographie $h$, le spectre de $\mathbf{C}_{s, t}$ sur $B_{\infty}(\mathcal{V})$ est formé des mêmes valeurs propres que le spectre de l'opérateur $\mathcal{C}_{s+t}$ sur $A_{\infty}(\mathcal{V})$, qui est égal, selon $(22)$, à la progression géométrique de premier terme $\mu_{0}=\widetilde{h}\left(z^{\star}\right)^{s+t}$ et de raison $\mu=(-1)^{k} \widetilde{h}\left(z^{\star}\right)^{2}$, où $k$ est la hauteur de l'homographie et $z^{\star}$ est l'unique point fixe de $h$ situé dans $\mathcal{V}$. Cependant, dans le spectre de $\mathbf{C}_{s, t}$, les valeurs propres sont multiples, et la valeur propre $\mu_{n}=\mu_{0} \mu^{n}$ indicée par $n$ a une multiplicité algébrique exactement égale à $n+1$. On remarque aussi que ce spectre peut être défini comme la réunion de spectres

$$
\operatorname{Sp} \mathbf{C}_{s, t}^{(h)}=\bigcup_{l \geq 0}(-1)^{k l} \operatorname{Sp} \mathcal{C}_{s+t+2 l}^{(h)}
$$

où la réunion est prise au sens des multi-ensembles, en préservant les multiplicités. C'est ce que montre le tableau suivant, où la référence à $h$ est omise :

$$
\begin{aligned}
& \mathrm{Sp} \mathbf{C}_{s, t}=\left\{\mu_{0}, \quad \mu_{1}, \quad \mu_{2}, \quad \ldots \quad \mu_{l}, \quad \ldots\right\},
\end{aligned}
$$

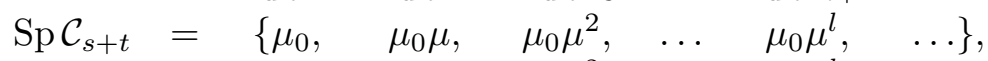

$$
\begin{aligned}
& (-1)^{k} \operatorname{Sp} \mathcal{C}_{s+t+2}=\left\{\begin{array}{lllll}
\mu_{0} \mu, & \mu_{0} \mu^{2}, & \ldots & \mu_{0} \mu^{l}, & \ldots\}
\end{array}\right\} \\
& (-1)^{2 k} \operatorname{Sp} \mathcal{C}_{s+t+4}=\left\{\begin{array}{llll}
\mu_{0} \mu^{2}, & \ldots & \mu_{0} \mu^{l}, & \ldots
\end{array},\right. \\
& (-1)^{l k} \operatorname{Sp} \mathcal{C}_{s+t+2 l}=\left\{\begin{array}{lll}
\mu_{0} \mu^{l}, & \ldots\}
\end{array} .\right.
\end{aligned}
$$

L'identité (46) permet d'obtenir la formule de la trace de l'opérateur $\mathbf{C}_{s, t}^{(h)}$ associé à une homographie $h$ de hauteur $k$,

$$
\operatorname{Tr} \mathbf{C}_{s, t}^{(h)}=\sum_{l \geq 0}(-1)^{k l} \operatorname{Tr} \mathcal{C}_{s+t+2 l}^{(h)}
$$

et donc par linéarité l'égalité (45), puis, en utilisant la définition (19) et la relation (20), l'identité (46) sur les déterminants de Fredholm, et enfin l'identité des spectres annoncée.

3.2. Les propriétés spectrales dominantes de $\mathbf{G}_{s, t}$ pour un couple $(s, t)$ de somme réelle. Dans le cas où les paramètres $s$ et $t$ ont une somme réelle, l'opérateur $\mathbf{G}_{s, t}$ a des propriétés spectrales dominantes qui sont essentiellement les mêmes que ceux de $\mathcal{G}_{s+t}$.

On montre d'abord directement pour l'opérateur $\mathbf{G}_{s, t}$ une propriété du type Perron-Frobenius, car il est de type $u_{0}$-positif. On désigne par $B_{\infty}^{\mathbb{R}}(\mathcal{V})$ les fonctions $F$ de $B_{\infty}(\mathcal{V})$ dont l'application diagonale $f$ est réelle sur $\mathcal{J}$, et par $K^{\prime}$ l'ensemble formé de la fonction nulle et des fonctions de $B_{\infty}^{\mathbb{R}}(\mathcal{V})$ dont l'application diagonale $f$ est positive mais non identiquement nulle sur $\mathcal{J}$. Le cône $K^{\prime}$ contient la fonction constante $u_{0}$ égale à 1 ; il est propre, reproductif, et d'intérieur non vide. L'opérateur $\mathbf{G}_{s, t}$ laisse stable $B_{\infty}^{\mathbb{R}}(\mathcal{V})$. 
Proposition 3. Soit $u_{0}=1$ la fonction constante égale à 1 . Pour $s$ et $t$ de somme réelle vérifiant $s+t>1$, l'opérateur $\mathbf{G}_{s, t}$ restreint à $B_{\infty}^{\mathbb{R}}(\mathcal{V})$ est $u_{0}$-positif par rapport au cône $K^{\prime}$.

Preuve. On adapte aisément la preuve par l'absurde que Mayer donne pour les opérateurs usuels, en utilisant la définition (26). Soit une fonction $F$ de $K^{\prime}$ pour laquelle pour tout entier $k$, il existe $u \in \mathcal{J}$ pour lequel $\mathbf{G}_{s, t}^{k}[F](u, u)=0$. Alors, pour toute homographie de hauteur $k$, on a $F(h(u), h(u))=f(h(u))=0$, ce qui entraîne, par continuité, que la restriction de $f$ à $\mathcal{J}$ est identiquement nulle. Donc $F$ est nécessairement nulle.

On peut donc appliquer le théorème de Krasnosel'skiı̌ [Kr], et conclure en utilisant la réalité du spectre de $\mathbf{G}_{s, t}$, qui démontre l'égalité des spectres de $\mathbf{G}_{s, t}$ et de sa restriction à $B_{\infty}^{\mathbb{R}}(\mathcal{V})$. Cette preuve n'est pas constructive car elle ne permet pas de relier directement les propriétés spectrales dominantes de $\mathbf{G}_{s, t}$ à celles de $\mathcal{G}_{s+t}$. C'est ce qui est établi dans le résultat suivant, qui constitue une généralisation presque complète du Théorème 2. Seule l'assertion (iii) du Théorème 5 est plus faible que l'assertion analogue (iv) du Théorème 2, et le recours aux espaces de Hilbert va permettre de renforcer cette assertion. Ce sera fait en 3.4, dans l'assertion (iii) du Théorème 7 .

THÉORÈme 5. Soient $s$ et $t$ deux complexes de somme réelle, vérifiant de plus

$$
s+t>1, \quad \Re(s) \geq 1, \quad \Re(t)>-1 .
$$

(i) L'opérateur $\mathbf{G}_{s, t}: B_{\infty}(\mathcal{V}) \rightarrow B_{\infty}(\mathcal{V})$ a une unique valeur propre dominante, égale à la valeur propre dominante $\lambda(s+t)$ de l'opérateur $\mathcal{G}_{s+t}$. Le vecteur propre dominant $F_{s, t}$ de $\mathbf{G}_{s, t}$ prolonge le vecteur propre dominant $f_{s+t}$ de $\mathcal{G}_{s+t}$ dans le sens suivant: $F_{s, t}$ est définie par

$$
F_{s, t}(u, v)=\int_{0}^{1} \beta_{t, s}(y) f_{s+t}(u+(v-u) y) d y,
$$

où $\beta_{t, s}$ est la densité $\beta$ classique,

$$
\beta_{t, s}(y)=\frac{\Gamma(s+t)}{\Gamma(s) \Gamma(t)} y^{t-1}(1-y)^{s-1},
$$

et $F_{s, t}$ vérifie $F_{s, t}(u, u)=f_{s+t}(u)$. Le vecteur propre dominant $F_{s, t}^{\star}$ de l'opérateur adjoint $\mathbf{G}_{s, t}^{\star}$ est égal au vecteur propre dominant $f_{s+t}^{\star}$ de $\mathcal{G}_{s+t}^{\star}$ dans le sens suivant : pour une fonction $F$ de $B_{\infty}(\mathcal{V})$ dont la restriction à la diagonale est $f$, on a $F_{s, t}^{\star}[F]=f_{s+t}^{\star}[f]$. Si $\mathbf{P}_{s, t}$ désigne la projection sur le sous-espace dominant définie par $\mathbf{P}_{s, t}=f_{s+t}^{\star} \otimes F_{s, t}$, alors $\mathbf{G}_{s, t}$ a la représentation $\mathbf{G}_{s, t}=\lambda(s+t) \mathbf{P}_{s, t}+\mathbf{N}_{s, t}$, où $\mathbf{P}_{s, t} \circ \mathbf{N}_{s, t}=\mathbf{N}_{s, t} \circ \mathbf{P}_{s, t}=0$. Pour une fonction $F \in B_{\infty}(\mathcal{V})$, on a 


$$
\mathbf{G}_{s, t}^{k}[F](u, v)=\lambda(s+t)^{k} f_{s+t}^{\star}[f] F_{s, t}(u, v)+\mathbf{N}_{s, t}^{k}[F](u, v)
$$

pour tout $k \geq 1$ et tout $(u, v)$ dans $\mathcal{V} \times \mathcal{V}$.

(ii) Le rapport spectral égal au rapport entre le rayon spectral de $\mathbf{N}_{s, t}$ et celui de $\mathbf{G}_{s, t}$ est strictement inférieur à 1 et ne dépend que de $s+t$; il est égal ̀̀

$$
\nu(s+t)=\frac{1}{\lambda(s+t)} \max (\lambda(s+t+2), \mu(s+t))
$$

où $\mu(s+t)$ est le rayon spectral de $\mathcal{N}_{s+t}$.

(iii) Pour toute constante $\alpha$ vérifiant $\nu(s+t)<\alpha<1$, on a

$$
\frac{\mathbf{G}_{s, t}^{k}[F](u, v)}{\lambda(s+t)^{k}}=f_{s+t}^{\star}[f] F_{s, t}(u, v)\left(1+O\left(\|F\| \alpha^{k}\right)\right)
$$

pour tout entier $k$ et pour toute fonction $F$ de $B_{\infty}(\mathcal{V})$ strictement positive sur la diagonale de $\mathcal{J} \times \mathcal{J}$. La constante du $O$ est uniforme sur $\mathcal{V} \times \mathcal{V}$ et dépend de $\alpha$.

R e m a r qu e. L'intégrale dans (48) n'est bien définie que pour $\Re(t)>0$. Sur un voisinage de $t=0$, on peut prolonger cette égalité en posant

$$
F_{s, t}(u, v)=f_{s+t}(u)+(v-u) \frac{t}{s+t} \int_{0}^{1} \beta_{t+1, s}(y) g_{s+t}(u+(v-u) y) d y,
$$

où $g_{s+t}$ est définie en fonction de $f_{s+t}$ par $f_{s+t}(z)=f_{s+t}(u)+(z-u) g_{s+t}(z)$.

Preuve du Théor ème 5. (i) et (ii) : Le fait que $\lambda(s+t)$ soit la valeur propre dominante et que $\nu(s+t)$ satisfasse (50) résulte de la décroissance stricte (40) de l'application $s \rightarrow \lambda(s)$.

En faisant le changement de variable $z=u+(v-u) y$ dans l'intégrale de (48), on obtient une autre expression de $F_{s, t}$,

$$
F_{s, t}(u, v)=\frac{\Gamma(s+t)}{\Gamma(s) \Gamma(t)} \int_{\gamma} f_{s+t}(z) \frac{(z-u)^{t-1}(v-z)^{s-1}}{(v-u)^{s+t-1}} d z,
$$

où $\gamma$ est le segment de droite $[u, v]$. La fonction à intégrer étant une fonction holomorphe de $z$, on remarque qu'on peut remplacer $\gamma$ par tout chemin simple reliant $u$ à $v$. On vérifie maintenant que l'égalité (53) définit plus généralement, à partir d'un vecteur propre $f$ de $\mathcal{G}_{s+t}$ relatif à $\lambda$, un vecteur propre $F$ de $\mathbf{G}_{s, t}$ relatif à $\lambda$. Si $F$ est définie par (53), le calcul de $\mathbf{G}_{s, t}[F]$ se ramène à évaluer l'expression de chaque $\mathbf{C}_{s, t}[F]$ associé à une homographie de hauteur 1, qui fait intervenir

$$
\frac{\widetilde{h}(u)^{s} \widetilde{h}(v)^{t}}{[h(v)-h(u)]^{s+t-1}} \int_{\delta} f(z)[z-h(u)]^{t-1}[h(v)-z]^{s-1} d z
$$


pour un chemin $\delta$ simple reliant $h(u)$ à $h(v)$. Comme l'intégrale ne dépend que des extrémités de $\delta$, on peut choisir pour $\delta$ l'image par $h$ d'un chemin $\gamma$ reliant simplement $u$ à $v$. Le changement de variable $z=h(w)$ dans (54), et les relations

$$
d z=h^{\prime}(w) d w=-\widetilde{h}(w)^{2} d w ; \quad h(a)-h(b)=-\widetilde{h}(a) \widetilde{h}(b)(a-b)
$$

valables pour $a$ et $b$ quelconques, permettent de réécrire (54) sous la forme

$$
\begin{aligned}
& \frac{1}{(v-u)^{s+t-1}} \int_{\gamma} \widetilde{h}(w)^{s+t} f \circ h(w)(w-u)^{t-1}(v-w)^{s-1} d w \\
& \quad=\frac{1}{(v-u)^{s+t-1}} \int_{\gamma} \mathcal{C}_{s+t}[f](w)(w-u)^{t-1}(v-w)^{s-1} d w .
\end{aligned}
$$

Pour $F$ définie à partir de $f$ par (53), on a donc établi la relation

$$
\mathbf{C}_{s, t}^{(h)}[F](u, v)=\frac{\Gamma(s+t)}{\Gamma(s) \Gamma(t)} \int_{\gamma} \mathcal{C}_{s+t}^{(h)}[f](w) \frac{(w-u)^{t-1}(v-w)^{s-1}}{(v-u)^{s+t-1}} d w,
$$

valable pour toute homographie de hauteur 1, et en sommant sur ces homographies, on obtient la relation

$$
\mathbf{G}_{s, t}[F]=\frac{\Gamma(s+t)}{\Gamma(s) \Gamma(t)} \int_{\gamma} \mathcal{G}_{s+t}[f](w) \frac{(w-u)^{t-1}(v-w)^{s-1}}{(v-u)^{s+t-1}} d w .
$$

Si donc $f$ est vecteur propre de $\mathcal{G}_{s+t}$ avec la valeur propre $\lambda$, alors $F$ est vecteur propre de $\mathbf{G}_{s, t}$ relatif à $\lambda$.

L'identité (12) de prolongement entre $\mathbf{G}_{s, t}$ et $\mathcal{G}_{s+t}$ permet alors d'obtenir l'expression de $F_{s, t}^{\star}$ en fonction de $f_{s+t}^{\star}$.

(iii) Il reste à évaluer alors la norme $\left\|\mathbf{N}_{s, t}^{k}\right\|$, ce qui se fait à l'aide du théorème du rayon spectral, par une preuve analogue à celle de la version faible du Théorème 2 .

3.3. Propriétés spectrales dominantes de l'opérateur $\mathbf{G}_{2, s}$ au voisinage de $s=0$. Comme pour les opérateurs de Ruelle-Mayer usuels, les propriétés spectrales dominantes se prolongent au voisinage de paramètres où elles sont bien définies, et ce, grâce à la théorie des perturbations. L'analyse de l'algorithme d'Euclide fait intervenir, via la Proposition 1, les opérateurs $\mathbf{G}_{2,-s}$. On va donc perturber l'opérateur $\mathbf{G}_{2,0}$ en $\mathbf{G}_{2,-s}$ sur l'espace $B_{\infty}(\mathcal{V})$, afin d'obtenir des propriétés spectrales dominantes de l'opérateur $\mathbf{G}_{2,-s}$ au voisinage de $s=0$. L'opérateur $\mathbf{G}_{2,0}: B_{\infty}(\mathcal{V}) \rightarrow B_{\infty}(\mathcal{V})$ a comme valeur propre dominante $\lambda(2)=1$. Le projecteur $\mathbf{N}_{2,0}$ a un rayon spectral égal ici au rapport spectral $\nu(2)$ de $\mathbf{G}_{2,0}$, qui compte tenu de (50), vérifie

$$
\nu(2)=\max (\mu(2), \lambda(4))=\mu(2) \approx 0.303663
$$


et est donc égal à la constante de Gauss-Kuz'min-Wirsing, puisque $\lambda(4) \approx$ 0.1994 (voir [DFV]).

Les conditions pour pouvoir perturber l'opérateur $\mathbf{G}_{2,0}$ dans l'espace $B_{\infty}(\mathcal{V})$ sont remplies : Il existe un voisinage complexe $\Omega$ de $s=0$ sur lequel (i) $\mathbf{G}_{2, s}: B_{\infty}(\mathcal{V}) \rightarrow B_{\infty}(\mathcal{V})$ est un opérateur borné, (ii) l'application $s \rightarrow \mathbf{G}_{2, s}$ définit une fonction analytique de $s$. La preuve de ces dernières assertions s'adapte facilement de la preuve de Faivre [Fa]. On peut alors utiliser les principes de la théorie de la perturbation pour décrire le comportement asymptotique des itérés de $\mathbf{G}_{2,-s}$ au voisinage de $s=0$. Le résultat suivant constitue ainsi une généralisation du Théorème 3 , et va permettre d'utiliser la Proposition 1.

ThÉORÈme 6 . Il existe un voisinage complexe $\Omega$ de $s=0$ où les propriétés spectrales dominantes de l'opérateur $\mathbf{G}_{2,0}: B_{\infty}(\mathcal{V}) \rightarrow B_{\infty}(\mathcal{V})$ se prolongent : sur ce voisinage, l'opérateur $\mathbf{G}_{2,-s}: B_{\infty}(\mathcal{V}) \rightarrow B_{\infty}(\mathcal{V})$ a une valeur propre dominante $\lambda(2-s)$ et un vecteur propre dominant $F_{2,-s}$ qui définissent des fonctions analytiques de $s$ ne s'annulant pas sur $\Omega$. Soit $\alpha$ une constante strictement inférieure à 1 et strictement supérieure à la constante de Gauss-Kuz'min-Wirsing $\mu(2)$. Il existe un voisinage $\Omega_{\alpha}$ de $s=0$ pour lesquels on a

$$
\frac{\mathbf{G}_{2,-s}^{k}[F](u, v)}{\lambda(2-s)^{k}}=f_{2-s}^{\star}[f] F_{2,-s}(u, v)\left(1+O\left(\|F\| \alpha^{k}\right)\right)
$$

pour tout entier $k$ et pour toute fonction $F$ de $B_{\infty}(\mathcal{V})$ strictement positive sur la diagonale de $\mathcal{J} \times \mathcal{J}$. La constante du $O$ est uniforme sur $\mathcal{V} \times \mathcal{V} \times \Omega_{\alpha}$ et dépend de $\alpha$.

Preu ve. La première assertion provient de la théorie des perturbations. En particulier, l'opérateur $\mathbf{N}_{2,-s}$ est bien défini sur le voisinage $\Omega$ et y définit une fonction analytique $s \rightarrow \mathbf{N}_{2,-s}$ de $s$. La norme $\left\|\mathbf{N}_{2,-s}\right\|$ définit alors une fonction continue de $s$ au voisinage de $s=0$. En $s=0$, d'après (56), le rayon spectral de $\nu(2)$ de $\mathbf{N}_{2,0}$ est égal à la constante de Wirsing $\mu(2)$. Le théorème du rayon spectral, rappelé en (37), affirme alors que pour tout nombre réel $\beta$ vérifiant $\mu(2)<\beta<1$, il existe un entier $k_{0}$ pour lequel la norme $\left\|\mathbf{N}_{2,0}^{k_{0}}\right\|$ de l'itéré de l'opérateur $\mathbf{N}_{2,0}$ dans $B_{\infty}(\mathcal{V})$ vérifie $\left\|\mathbf{N}_{2,0}^{k_{0}}\right\| \leq \beta^{k_{0}}$. Par ailleurs, on pose

$$
B=\max \left\{\left\|\mathbf{N}_{2,0}^{j}\right\| \mid 0 \leq j \leq k_{0}-1\right\} .
$$

Alors, par continuité, si on choisit $\gamma$ et $C$ vérifiant $\beta<\gamma<1$ et $C>B$, il existe un voisinage $\Omega$ de $s=0$ pour lequel on a

$$
\left\|\mathbf{N}_{2,-s}^{k_{0}}\right\| \leq \gamma^{k_{0}} \quad \text { et } \quad \max \left\{\left\|\mathbf{N}_{2,0}^{j}\right\| \mid 0 \leq j \leq k_{0}-1\right\} \leq C
$$

pour tout $s$ dans $\Omega$. Sur ce même voisinage et pour tout indice $k$, on déduit

$$
\left\|\mathbf{N}_{2,-s}^{k}\right\| \leq C \gamma^{k_{0}\left[k / k_{0}\right]} \leq A \gamma^{k}
$$


où la constante $A$ dépend de $\gamma$ mais est indépendante de $k$ et de $s \in \Omega$. Par ailleurs, en $s=0$, la valeur propre dominante $\lambda(2-s)$ est égale à 1 . Si on choisit alors $\alpha$ vérifiant $\gamma<\alpha<1$ et si on restreint au besoin le voisinage $\Omega$ de $s=0$, on obtient sur ce voisinage l'inégalité

$$
\frac{\left\|\mathbf{N}_{2, s}^{k}\right\|}{\lambda^{k}(2-s)} \leq A \alpha^{k}
$$

pour tout $s$ de $\Omega$. Finalement, sur $\Omega$, pour toute fonction $F$ de $B_{\infty}(\mathcal{V})$, et pour tout entier $k$, on a

$$
\left\|\frac{\mathbf{G}_{2,-s}^{k}[F]}{\lambda(2-s)^{k}}-f_{2-s}^{\star}[f] F_{2,-s}\right\| \leq A\|F\| \alpha^{k} .
$$

3.4. Représentation intégrale des opérateurs de Ruelle-Mayer à deux variables. La structure d'espace de Hilbert introduite par Babenko puis Mayer s'adapte bien aux opérateurs $\mathbf{G}_{s, t}$, du moins lorsque $s$ et $t$ vérifient les conditions

$$
\Re(s)>0, \quad \Re(t)>0, \quad \Re(s+t)>1 .
$$

Cette structure joue le même rôle que dans la section précédente; elle permet de montrer que ces opérateurs sont diagonalisables, et d'évaluer précisément leur norme. Comme précédemment, ces espaces de Hilbert sont fortement comparables aux espaces de Banach initiaux, on peut "transporter" la plupart des résultats obtenus dans les espaces de Banach initiaux, ce qui permet une évaluation fine des termes-reste.

On considère les demi-plans $\mathcal{P}_{\varrho}=\{z \mid \Re(z)>\varrho\}, \mathcal{P}:=\mathcal{P}_{-1 / 2}$ et l'espace de Hilbert $\mathbf{H}_{s, t}$ formé des fonctions $F$ holomorphes sur $\mathcal{P} \times \mathcal{P}$, bornées sur $\mathcal{P}_{\varrho} \times \mathcal{P}_{\varrho}$ pour tout $\varrho>-1 / 2$ et admettant une représentation intégrale de la forme

$$
F(u, v)=\int_{0}^{\infty} \int_{0}^{\infty} x^{(s-1) / 2} y^{(t-1) / 2} e^{-(u x+v y)} \Phi(x, y) \frac{d x d y}{e^{x+y}-1}
$$

où $\Phi$ est de carré intégrable par rapport à la mesure $d m(x, y)$ de densité $1 /\left(e^{x+y}-1\right)$. Compte tenu de la relation valable quand $\sigma:=\Re(s)$ et $\tau:=\Re(t)$ vérifient (58),

$$
\int_{0}^{\infty} \int_{0}^{\infty} x^{\sigma-1} y^{\tau-1} \frac{d x d y}{e^{x+y}-1}=\Gamma(\sigma) \Gamma(\tau) \zeta(\sigma+\tau),
$$

la fonction $x^{(s-1) / 2} y^{(t-1) / 2}$ est de carré intégrable par rapport à la mesure $d m(x, y)$ de densité $1 /\left(e^{x+y}-1\right)$. Il est alors légitime de définir la norme $|\cdot|_{\langle s, t\rangle}$ sur cet espace $\mathbf{H}_{s, t}$ par

$$
|F|_{\langle s, t\rangle}^{2}=\int_{0}^{\infty} \int_{0}^{\infty}|\Phi(x, y)|^{2} \frac{d x d y}{e^{x+y}-1} .
$$


Le résultat suivant constitue une généralisation du Théorème 1 , et de la relation (iv) du Théorème 2 , dont on n'avait obtenu jusqu'à présent qu'une forme faible. Il permet d'évaluer les vitesses de convergence en fonction du rapport spectral.

ThÉORÈme 7 . Soient $s$ et $t$ deux paramètres complexes vérifiant

$$
\Re(s)>0, \quad \Re(t)>0, \quad \Re(s+t)>1 .
$$

(i) L'opérateur $\mathbf{G}_{s, t}: \mathbf{H}_{s, t} \rightarrow \mathbf{H}_{s, t}$ est isomorphe à un opérateur intégral; il est normal et est donc diagonalisable sur l'espace $\mathbf{H}_{s, t}$ dans une base orthonormale. De plus, pour s et $t$ réels, l'opérateur $\mathbf{G}_{s, t}$ est auto-adjoint.

(ii) Si $s$ et $t$ vérifient $\Re(s) \geq 1, \Re(t) \geq 1$, les deux normes $\|\cdot\|$ et $|\cdot|_{\langle s, t\rangle}$ sont quasi-équivalentes dans le sens suivant : La fonction $\mathbf{G}_{s, t}[F]$ est élément de $\mathbf{H}_{s, t}$ dès que la fonction $F$ est élément de $B_{\infty}(\mathcal{V})$ et il existe deux constantes $A(s, t)$ et $B(s, t)$ pour lesquelles on a la double inégalité

$$
\begin{gathered}
\|F\| \leq A(s, t)|F|_{\langle s, t\rangle} \quad \text { pour } F \in \mathbf{H}_{s, t}, \\
\left|\mathbf{G}_{s, t}[F]\right|_{\langle s, t\rangle} \leq B(s, t)\|F\| \quad \text { pour } F \in B_{\infty}(\mathcal{V}) .
\end{gathered}
$$

(iii) Si s et $t$ sont réels et vérifient $s \geq 1, t \geq 1$, et si $F$ est une fonction de $B_{\infty}(\mathcal{V})$ strictement positive sur la diagonale de $\mathcal{J} \times \mathcal{J}$, on a

$$
\frac{\mathbf{G}_{s, t}^{k}[F](u, v)}{\lambda(s+t)^{k}}=f_{s+t}^{\star}[f] F_{s, t}(u, v)\left(1+O\left(\|F\| \nu(s+t)^{k}\right)\right)
$$

pour tout entier $k$. La constante du $O$ est uniforme $\operatorname{sur} \mathcal{V} \times \mathcal{V}$ et dépend de $\alpha$ et de $s, t$.

Preuve. (i) Sur l'espace $\mathbf{H}_{s, t}$, l'opérateur $\mathbf{G}_{s, t}$ s'exprime sous la forme

$$
\mathbf{G}_{s, t}[F](u, v)=\int_{0}^{\infty} \int_{0}^{\infty} X^{(s-1) / 2} Y^{(t-1) / 2} e^{-(u X+v Y)} \mathbf{K}_{s, t}[\Phi](X, Y) \frac{d X d Y}{e^{X+Y}-1}
$$

où $\mathbf{K}_{s, t}$ est un opérateur intégral faisant intervenir le produit des fonctions de Bessel d'indice $s-1$ et d'indice $t-1$,

$$
\mathbf{K}_{s, t}[\Phi](X, Y)=\int_{0}^{\infty} \int_{0}^{\infty} J_{s-1}(2 \sqrt{x X}) J_{t-1}(2 \sqrt{y Y}) \Phi(x, y) \frac{d x d y}{e^{x+y}-1} .
$$

Ainsi, restreint à cet espace $\mathbf{H}_{s, t}$, l'opérateur $\mathbf{G}_{s, t}$ est isomorphe à un opérateur intégral dont le noyau est le produit des fonctions de Bessel d'indice $s-1$ et $t-1$. Ceci prouve (i).

(ii) On peut généraliser (28) en (59) : grâce à l'inégalité de CauchySchwarz, pour tout couple $(s, t)$ vérifiant $\Re(s)>0, \Re(t)>0$, il existe une constante $A(s, t)$ pour laquelle

$$
|F(u, v)| \leq A(s, t)|F|_{\langle s, t\rangle} \quad \text { pour tout }(u, v) \in \mathcal{V} \times \mathcal{V} .
$$


Pour deux nombres complexes $s$ et $t$ vérifiant $\Re(s) \geq 2$ et $\Re(t) \geq 2$, l'espace $\mathbf{H}_{s, t}$ est identique à l'espace $\widehat{\mathbf{H}}_{s, t}$ formé des fonctions holomorphes sur $\mathcal{P} \times \mathcal{P}$, bornées sur tout $\mathcal{P}_{\varrho} \times \mathcal{P}_{\varrho}(\varrho>-1 / 2)$, et vérifiant

$$
\int_{\widetilde{\mathcal{B}}}\left|x_{u}\right|^{\sigma-2}\left|x_{v}\right|^{\tau-2}|F(u, v)|^{2} d x_{u} d y_{u} d x_{v} d y_{v}<\infty .
$$

Ici, on pose $\sigma=\Re(s), \tau=\Re(t), u=x_{u}+i y_{u}, v=x_{v}+i y_{v}$ et on désigne par $\widetilde{\mathcal{B}}$ la réunion des deux domaines

$$
\widetilde{\mathcal{B}}:=\{(u, v) \mid-1 / 2<\Re(u) \leq 0\} \cup\{(u, v) \mid-1 / 2<\Re(v) \leq 0\} .
$$

Le théorème de Plancherel permet de généraliser (30) en

$$
\begin{aligned}
& |F|_{\langle s, t\rangle}^{2} \\
& \quad=\frac{2^{\sigma-2} 2^{\tau-2}}{\pi^{2} \Gamma(\sigma-2) \Gamma(\tau-2)} \int_{\tilde{\mathcal{B}}}\left|x_{u}\right|^{\sigma-2}\left|x_{v}\right|^{\tau-2}|F(u, v)|^{2} d x_{u} d y_{u} d x_{v} d y_{v} .
\end{aligned}
$$

Ceci permet de prouver que, pour $\Re(s) \geq 2, \Re(t) \geq 2$, la fonction $\mathbf{G}_{s, t}[F]$ est élément de $\mathbf{H}_{s, t}$ dès que la fonction $F$ est élément de $B_{\infty}(\mathcal{V})$. On utilise pour cela deux relations qui généralisent (31) et (32) : la première se déduit de (62), pour $\Re(s) \geq 2$ et $\Re(t) \geq 2$,

$$
\left|\mathbf{G}_{s, t}[F]\right|_{\langle s, t\rangle} \leq\|F\| \sum_{m \geq 1}\left|\frac{1}{(m+u)^{s}(m+v)^{t}}\right|_{\langle s, t\rangle},
$$

et la seconde s'obtient en calculant directement l'élément $\Phi$ associé à chaque fonction de $\mathbf{H}_{s_{0}, t_{0}}$ de la forme $(u, v) \rightarrow 1 /\left((m+u)^{s}(m+v)^{t}\right)$ lorsque $\sigma:=$ $\Re(s), \sigma_{0}:=\Re\left(s_{0}\right), \tau:=\Re(t)$ et $\tau_{0}:=\Re\left(t_{0}\right)$ vérifient $2 \sigma-\sigma_{0}>0,2 \tau-\tau_{0}>0$, et $2(\sigma+\tau)-\left(\sigma_{0}+\tau_{0}\right)>1$. On a alors

$$
\sum_{m \geq 1}\left|\frac{1}{(m+u)^{s}(m+v)^{t}}\right|_{\left\langle s_{0}, t_{0}\right\rangle} \leq \frac{1}{|\Gamma(s) \Gamma(t)|} D\left(2 \sigma-\sigma_{0}, 2 \tau-\tau_{0}\right)
$$

avec

$$
D(\alpha, \beta)=(\alpha+\beta) \Gamma(\alpha) \Gamma(\beta)^{1 / 2} \widetilde{\zeta}\left(\frac{\alpha+\beta+1}{2}\right),
$$

où $\widetilde{\zeta}$ représente la somme des termes d'indice impair de la fonction $\zeta$. Cette inégalité s'applique en particulier pour $s=s_{0}, t=t_{0}$ et $s$ et $t$ vérifiant (58).

Pour les autres valeurs de $s$ et $t$ considérées ici, $1 \leq \Re(s)<2$ et $1 \leq \Re(t)<2$, on peut redéfinir l'espace $\mathbf{H}_{s, t}$ en fonction des espaces $\widehat{\mathbf{H}}_{s, t}$ : l'espace $\mathbf{H}_{s, t}$ est identique à l'espace formé par les fonctions $F$ holomorphes sur $\mathcal{P}$, bornées sur tout $\mathcal{P}_{\varrho}(\varrho>-1 / 2)$, s'annulant ainsi que leur dérivée $\partial F / \partial v$ pour $\Re(v) \rightarrow \infty$, et dont la dérivée $\partial^{2} F / \partial v \partial u$ appartient à 
l'espace $\widehat{\mathbf{H}}_{s+2, t+2}$. En utilisant l'expression de $\left(\partial^{2} / \partial v \partial u\right) \mathbf{G}_{s, t}[F]$ en fonction d'opérateurs $\mathbf{G}_{s^{\prime}, t^{\prime}}$ généralisant (28),

$$
\begin{aligned}
\frac{\partial^{2}}{\partial v \partial u} \mathbf{G}_{s, t}[F]= & s t \mathbf{G}_{s+1, t+1}[F] \\
& +t \mathbf{G}_{s+2, t+1}\left[\frac{\partial F}{\partial u}\right]+s \mathbf{G}_{s+1, t+2}\left[\frac{\partial F}{\partial v}\right]+\mathbf{G}_{s+2, t+2}\left[\frac{\partial^{2} F}{\partial u \partial v}\right],
\end{aligned}
$$

et les relations (63) et (64), on démontre alors que, pour une fonction $F$ de $B_{\infty}(\mathcal{V})$, la fonction $\mathbf{G}_{s, t}[F]$ est élément de $\mathbf{H}_{s, t}$.

(iii) On fait une démonstration analogue à celle de l'assertion (iv) du Théorème 2 .

La première section a montré comment les objets centraux dans l'analyse en moyenne des deux algorithmes étudiés s'expriment en fonction des itérés des opérateurs $\mathbf{G}_{s, t}$. Dans les sections 2 et 3 , nous avons étudié les propriétés spectrales dominantes de ces opérateurs. Il reste donc à utiliser les résultats des sections précédentes afin de décrire le comportement asymptotique des deux algorithmes étudiés.

4. Opérateurs de Ruelle-Mayer généralisés et convergence de la loi de $\log Q_{k}$ vers la loi normale. Ordinairement, l'étude de cette convergence se fait à l'aide de la fonction caractéristique. C'est la démarche utilisée par Philipp et Morita; ils observent que la fonction $\log Q_{k}$ est peu différente de la somme des $k$ variables $\log U^{l}$ pour $l \leq k$, où $U^{l}$ est le $l$-ème itéré de l'opérateur de décalage $U$. Cette démarche présente un inconvénient majeur : comme on travaille seulement avec une approximation de $\log Q_{k}$, on ne parvient à préciser complètement le comportement asymptotique de la variable $\log Q_{k}$. Par ailleurs, ces techniques ne permettent pas de traiter a priori le cas d'une densité quelconque.

Nous proposons de travailler directement avec la variable $\log Q_{k}$, et, suite à des travaux récents de Hwang, nous choisissons l'outil de la série génératrice des moments $M_{k}$. La thèse de Hwang toute entière montre l'application d'un tel outil, lorsque la distribution-limite est gaussienne, avec une moyenne et une variance ayant le même ordre de grandeur. Nous utilisons ici deux principaux ingrédients :

(a) Un théorème dû à Hwang [Hw], rappelé ci-dessous en Théorème 8 , qui donne des conditions suffisantes sur la suite $M_{k}$ sous lesquelles il y a convergence vers la loi normale. Ces conditions consistent essentiellement en l'approximation de $M_{k}(s)$ par une grande puissance, avec l'existence d'un reste uniforme au voisinage de l'origine.

(b) Via l'expression de $M_{k}(s)$ obtenue dans la Proposition 1, ces conditions sont remplies grâce au comportement spectral dominant des opérateurs 
$\mathbf{G}_{2,-s}$ au voisinage de $s=0$, que nous avons établi dans le Théorème 6 , et que nous adaptons au cadre précis dans la Proposition 4.

4.1. Conditions suffisantes de convergence vers la loi normale. Soit $\Phi(x)$ $=(2 \pi)^{-1} \int_{-\infty}^{x} e^{-t^{2} / 2} d t$ la fonction de répartition gaussienne. Le théorème de Hwang sur les séries génératrices des moments est le suivant :

ThÉORÈme 8 [Hwang]. Soit une suite $\Omega_{k}$ de variables aléatoires, dont les séries génératrices des moments admettent l'expression asymptotique suivante:

$$
M_{k}(s):=E\left[\exp \left(s \Omega_{k}\right)\right]=\exp V_{k}(s)\left(1+O\left(1 / W_{k}\right)\right)
$$

le $O$ étant uniforme sur un disque complexe fermé $|s| \leq s_{0}, s_{0}>0$ où :

(i) $V_{k}(s)=U(s) \phi_{k}+V(s)$, avec $U(s)$ et $V(s)$ analytiques pour $|s| \leq s_{0}$ $(U(0)=V(0)=0)$ et indépendantes de $k$; de plus, $U^{\prime \prime}(0) \neq 0$,

(ii) $\phi_{k}$ et $W_{k}$ tendent vers $\infty$ quand $k$ tend vers $\infty$.

On pose $\mu_{k}=U^{\prime}(0) \phi_{k}, \sigma_{k}^{2}=U^{\prime \prime}(0) \phi_{k}$, et $S_{k}=\min \left(\sqrt{\phi_{k}}, W_{k}\right)$, et on considère la série entière $R(t):=\sum_{m \geq 3} r_{m} t^{m}$ où le coefficient $r_{m}$ est le coefficient de $w^{m-2}$ dans l'expression

$$
\frac{-1}{m} U^{\prime \prime}(w)\left(\frac{U(w)-U^{\prime}(0)}{U^{\prime \prime}(0) w}\right)^{-m}
$$

Alors, la moyenne et la variance de $\Omega_{k}$ vérifient :

$$
E\left[\Omega_{k}\right]=\mu_{k}+V^{\prime}(0)+O\left(\frac{1}{W_{k}}\right), \quad \operatorname{Var}\left[\Omega_{k}\right]=\sigma_{k}^{2}+V^{\prime \prime}(0)+O\left(\frac{1}{W_{k}}\right) .
$$

De plus, la distribution de $\Omega_{k}$ est asymptotiquement gaussienne : la fonction de répartition

$$
P_{k}(x):=\operatorname{Pr}_{f}\left[\frac{\log Q_{k}-A k}{\sqrt{B k}}<x\right]
$$

converge vers la fonction de répartition gaussienne $\Phi(x)$ sous la forme

$$
P_{k}(x)=\Phi(x)+O\left(1 / S_{k}\right)
$$

uniformément en $x$ décrivant $\mathbb{R}$, quand $k$ tend vers $\infty$. Enfin, il y a un résultat de grandes déviations : les deux fonctions de répartition vérifient pour $x>0, x=o\left(S_{k}\right)$, et $t=x / \sigma_{k}$ les deux relations

$$
\begin{aligned}
& \frac{1-P_{k}(x)}{1-\Phi(x)}=\exp \left[\phi_{k} R(t)\right]\left(1+O\left(\frac{x}{S_{k}}\right)\right), \\
& \frac{P_{k}(-x)}{\Phi(-x)}=\exp \left[\phi_{k} R(-t)\right]\left(1+O\left(\frac{x}{S_{k}}\right)\right) .
\end{aligned}
$$

Preuve. Elle est adaptée de la preuve de Hwang et utilise essentiellement l'inégalité de Berry-Esseen qui permet de majorer l'écart entre les 
fonctions de répartition $F$ et $G$ en fonction de l'écart entre les fonctions caractéristiques $\varphi$ et $\gamma$ :

$$
\sup _{x \in \mathbb{R}}|F(x)-G(x)| \leq b \int_{-T}^{T}\left|\frac{\varphi(t)-\gamma(t)}{t}\right| d t+r(b) \frac{A}{T}
$$

où $T$ est un nombre positif quelconque, $b$ vérifie $b \geq 1 /(2 \pi)$ et $r(b)$ est une fonction de $b$.

Ici, on considère la fonction caractéristique $\varphi_{k}$ de la variable $\left(\Omega_{k}-\right.$ $\left.\mu_{k}\right) / \sigma_{k}$, et on choisit $T_{k}=c \sigma_{k}$, pour une constante $c>0$ suffisamment petite. On va prouver que

$$
I_{k}=\int_{-T_{k}}^{T_{k}} \frac{1}{|t|}\left|\varphi_{k}(t)-\exp \left(\frac{-t^{2}}{2}\right)\right| d t=O\left(\frac{1}{\sigma_{k}}+\frac{1}{W_{k}}\right),
$$

ce qui permettra de montrer la première partie du théorème. La fonction caractéristique $\varphi_{k}$ s'exprime en fonction de $M_{k}$

$$
\varphi_{k}(t)=\exp \left(-i t \frac{\mu_{k}}{\sigma_{k}}\right) M_{k}\left(\frac{i t}{\sigma_{k}}\right),
$$

et on réécrit $M_{k}$ sous la forme $M_{k}(s)=R_{k}(s)\left(1+a_{k}(s)\right)$, avec

$$
R_{k}(s):=\exp \left[U(s) \phi_{k}+V(s)\right], \quad a_{k}(s)=O\left(1 / W_{k}\right) .
$$

Comme les dérivées $\left|U^{\prime \prime \prime}\right|$ et $\left|V^{\prime}\right|$ sont bornées au voisinage de l'origine,

$$
\exp \left(-i t \frac{\mu_{k}}{\sigma_{k}}\right) R_{k}\left(\frac{i t}{\sigma_{k}}\right)=\exp \left(\frac{-t^{2}}{2}\right) \exp f(t),
$$

avec

$$
|f(t)| \leq \frac{\alpha|t|+\beta\left|t^{3}\right|}{\sigma_{k}} \leq \delta+\frac{t^{2}}{4}
$$

pour des constantes $\alpha, \beta, \delta$ et pour $|t| \leq T_{k}$. D'autre part, la fonction $a_{k}$ est analytique sur un voisinage de 0 et y est un $O\left(1 / W_{k}\right)$. Alors, en utilisant la formule de Cauchy dans un voisinage de l'origine, on déduit que $a_{k}^{\prime}(s)$ est elle-même un $O\left(1 / W_{k}\right)$ sur un voisinage de 0 ; comme de plus $a_{k}(s)$ est nulle en $s=0$, on déduit

$$
\left|a_{k}(s)\right| \leq \frac{\varrho}{W_{k}}|s|
$$

pour une constante $\varrho$ sur un petit voisinage $|s| \leq \nu$ de 0 . Finalement,

$$
\begin{aligned}
\frac{1}{|t|}\left|\varphi_{k}(t)-\exp \left(\frac{-t^{2}}{2}\right)\right| & \\
& \leq \frac{\alpha+\beta t^{2}}{\sigma_{k}} \exp \left(\delta+\frac{-t^{2}}{4}\right)+\left|\frac{1}{t} R_{k}\left(\frac{i t}{\sigma_{k}}\right) a_{k}\left(\frac{i t}{\sigma_{k}}\right)\right| .
\end{aligned}
$$


En intégrant le premier terme, on obtient un terme en

$$
O\left(\frac{1}{\sigma_{k}}\right) \int_{-T_{k}}^{T_{k}}\left(1+t^{2}\right) \exp \left(\frac{-t^{2}}{4}\right) d t=O\left(\frac{1}{\sigma_{k}}\right) .
$$

Pour le second terme, on met à part le segment $\left[-\nu \sigma_{k}, \nu \sigma_{k}\right]$ pour lequel, grâce à (65) et (66), l'intégrale donne un terme en $O\left(1 / W_{k}\right)$. Sur les deux autres segments $\left[-T_{k},-\nu \sigma_{k}\right]$ et $\left[\nu \sigma_{k}, T_{k}\right]$, la fonction à intégrer est majorée, grâce à (65), par

$$
O\left(\frac{1}{\sigma_{k} W_{k}}\right) \exp \left(\frac{-t^{2}}{4}\right),
$$

et l'intégrale donne un terme en $O\left(1 /\left(\sigma_{k} W_{k}\right)\right)$. Finalement, l'intégrale de Berry-Esseen a bien la forme cherchée.

La deuxième partie du théorème utilise la fonction génératrice des cumulants

$$
C_{k}(s):=\log M_{k}(s)=U(s) \phi_{k}+V(s)+\log \left[1+a_{k}(s)\right] .
$$

En effet, $E\left[\Omega_{k}\right]$ et $\operatorname{Var}\left[\Omega_{k}\right]$ sont respectivement égaux à $C_{k}^{\prime}(0)$ et $C_{k}^{\prime \prime}(0)$. Par ailleurs, la formule de Cauchy appliquée à la fonction analytique $\log \left[1+a_{k}(s)\right]$ permet de montrer que les deux premières dérivées de cette fonction en 0 sont des $O\left(1 / W_{k}\right)$. On obtient ainsi l'expression de $E\left[\Omega_{k}\right]$ et de $\operatorname{Var}\left[\Omega_{k}\right]$.

La preuve de la dernière assertion sur les grandes déviations est plus technique et est ici omise.

4.2. Comportement asymptotique des itérés de $\mathbf{G}_{2,-s}$ au voisinage de $s=0$. On peut immédiatement utiliser ce théorème dès qu'on obtient un comportement dominant de l'opérateur $\mathbf{G}_{2,-s}$ sur un voisinage de $s=0$. Les Théorèmes 3 et 6 nous fournissent un tel résultat pour les opérateurs $\mathcal{G}_{2-s}$ ou $\mathbf{G}_{2,-s}$, et la Proposition 1 permet de conclure, pourvu que l'on ait préalablement adapté l'espace des fonctions sur lequel on travaille. C'est ce qu'on fait maintenant :

Proposition 4. Soit $\alpha$ une constante strictement inférieure à 1 et strictement supérieure à la constante de Gauss-Kuz'min-Wirsing $\mu(2)$. Pour toute fonction $f$ analytique et strictement positive sur $\overline{\mathcal{I}}=[0,1]$, il existe un voisinage complexe $\Omega_{f} \subset \Omega$ et une fonction $p$ analytique sur $\Omega_{f}$, et ne s'annulant pas sur $\Omega_{f}$ pour lesquels on a la relation suivante faisant intervenir $F$ définie par $F(u, v):=f(u)$ :

$$
\mathbf{G}_{2,-s}^{k}[F](u, v)=\lambda(2-s)^{k} p(s) F_{2,-s}(u, v)\left(1+O\left(\alpha^{k}\right)\right),
$$

pour tout $k$ suffisamment grand, uniformément en $(u, v) \in \overline{\mathcal{I}} \times \overline{\mathcal{I}}$ et en $s \in \Omega_{f}$. 
Preuve. Il s'agit d'adapter le résultat du Théorème 6 au problème. Dans le cas où $f$ est analytique sur $\overline{\mathcal{I}}$, elle se prolonge à une fonction analytique, notée encore $f$, sur un rectangle ouvert de la forme $\mathcal{R}:=]-\varrho, 1+\varrho[\times$ ]$-\varrho, \varrho\left[\right.$ avec $\varrho>0$. Or, à tout $\varrho>0$, on peut associer un entier $k_{1}$ pour lequel

$$
\bigcup_{|h|=k} h(\mathcal{V}) \subset \mathcal{R}_{\varrho} \quad \text { pour tout } k \geq k_{0} .
$$

En effet, pour une hauteur $k$ fixée, le disque $h(\mathcal{V})$ de plus grand rayon est celui qui est lié à l'homographie $h_{\mathbf{m}}$ avec tous les $m_{i}$ égaux à 1 . Les continuants associés sont alors les nombres de Fibonacci $\phi_{k}$. On en déduit donc, par un calcul facile, que tous les disques $h(\mathcal{V})$ de hauteur $k$ sont situés dans le rectangle $\mathcal{R}_{\varrho}$ dès que $\varrho \geq 4 / \phi_{k}^{2}$, ce qui démontre (68). On considère alors, pour $F$ définie à partir de $f$ par $F(u, v):=f(u)$, la fonction

$$
L_{s}:=\mathbf{G}_{2,-s}^{k_{1}}[F]
$$

c'est un élément de $B_{\infty}(\mathcal{V})$, d'application diagonale $l_{s}:=\mathcal{G}_{2-s}^{k_{1}}[f]$, à qui on peut appliquer le Théorème 6 :

$$
\left\|\frac{\mathbf{G}_{2,-s}^{k}\left[L_{s}\right]}{\lambda(2-s)^{2 k}}-f_{2-s}^{\star}\left[l_{s}\right] F_{2,-s}\right\| \leq A\left\|L_{s}\right\| \alpha^{k}
$$

sur le voisinage $\Omega$ de $s=0$. Posant alors, par définition,

$$
p(s):=\frac{1}{\lambda(2-s)^{k_{1}}} f_{2-s}^{\star}\left[l_{s}\right],
$$

on obtient une fonction $p$ analytique sur ce même voisinage. Cette fonction $p$ n'est pas nulle en $s=0$, puisque, d'après (42),

$$
p(0)=f_{2}^{\star}\left[l_{0}\right]=f_{2}^{\star}\left[\mathcal{G}_{2}^{k_{1}}[f]\right]=f_{2}^{\star}[f]=\int_{0}^{1} f(x) d x,
$$

qui est strictement positive par hypothèse. Quitte à restreindre $\Omega$ en $\Omega_{f}$, la fonction $p$ est minorée en module par une constante strictement positive. Puisque $F_{2,0}(u, v)=f_{2}(u)$, il existe aussi un voisinage de $s=0$ pour lequel $F_{2,-s}(u, v)$ est minorée en module par une constante strictement positive, uniformément en $(u, v) \in \overline{\mathcal{J}} \times \overline{\mathcal{J}}$. Par ailleurs, sur ce même voisinage, puisque $\mathbf{G}_{2, s}$ est borné sur $B_{\infty}(\mathcal{V})$, la norme $\left\|L_{s}\right\|$ définit une fonction continue de $s$, qui est donc bornée. En remplaçant alors $L_{s}$ par sa valeur, on déduit finalement de (69) la relation cherchée pour tout $k \geq k_{1}$.

4.3. La convergence de $\log Q_{k}$ vers la loi normale. Les deux résultats précédents permettent alors de déduire aisément le résultat suivant, qui constitue la conclusion du premier axe de notre travail. 
THÉORÈme 9. Soit $f$ une densité analytique et strictement positive sur $\overline{\mathcal{I}}$. La moyenne et la variance de la variable aléatoire $\log Q_{k}$ par rapport à la mesure de densité $f$ vérifient les deux relations

$$
E_{f}\left[\log Q_{k}\right]=A k+C_{f}+O\left(\alpha^{k}\right) \text { et } \operatorname{Var}_{f}\left[\log Q_{k}\right]=B k+D_{f}+O\left(\alpha^{k}\right) .
$$

Ici, les constantes $A$ et $B$ sont indépendantes de la densité $f$ et s'expriment en fonction des dérivées de la fonction $U(s):=\log \lambda(2-s)$ en $s=0$ :

$$
\begin{aligned}
& A=[\log \lambda(2-s)]_{(s=0)}^{\prime}=-\lambda^{\prime}(2), \\
& B=[\log \lambda(2-s)]_{(s=0)}^{\prime \prime}=\lambda^{\prime \prime}(2)-\lambda^{\prime}(2)^{2},
\end{aligned}
$$

tandis que les constantes $C$ et $D$, tout comme les constantes des $O$, dépendent de $f$. On peut choisir pour $\alpha$, tout nombre strictement inférieur à 1 , et majorant strictement la constante de Gauss-Kuz'min-Wirsing $\mu(2) \approx$ 0.303663. La distribution de la variable aléatoire $\log Q_{k}$ par rapport à la mesure de densité $f$ est asymptotiquement gaussienne : la fonction de répartition

$$
P_{k}(x):=\operatorname{Pr}_{f}\left[\frac{\log Q_{k}-A k}{\sqrt{B k}}<x\right]
$$

converge vers la fonction de répartition gaussienne $\Phi(x)$ dans le sens suivant :

$$
P_{k}(x)=\Phi(x)+O(1 / \sqrt{k})
$$

uniformément en $x$ décrivant $\mathbb{R}$, quand $k$ tend vers $\infty$. Enfin, les deux fonctions de répartition vérifient pour $x>0, x=o\left(S_{k}\right)$, et $t=x / \sigma_{k}$,

$$
\begin{aligned}
\frac{1-P_{k}(x)}{1-\Phi(x)} & =\exp [k R(t)]\left(1+O\left(\frac{x}{\sqrt{k}}\right)\right), \\
\frac{P_{k}(-x)}{\Phi(-x)} & =\exp [k R(-t)]\left(1+O\left(\frac{x}{\sqrt{k}}\right)\right),
\end{aligned}
$$

où la série entière $R(t):=\sum_{m \geq 3} r_{m} t^{m}$ a pour coefficient $r_{m}$ le coefficient de $w^{m-2}$ dans l'expression

$$
\frac{-1}{m} U^{\prime \prime}(w)\left(\frac{U(w)-U^{\prime}(0)}{U^{\prime \prime}(0) w}\right)^{-m}
$$

avec $U(s):=\log \lambda(2-s)$.

Preuve. On applique le Théorème 8 de Hwang en utilisant l'expression (15), donnée dans la Proposition 1, de la série génératrice des moments en fonction de l'opérateur $\mathbf{G}_{2,-s}$ et le comportement spectral dominant de cet opérateur $\mathbf{G}_{2,-s}$ au voisinage de $s=0$ obtenu en (67) dans la Proposition 4. 
Les Propositions 1 et 4 permettent d'écrire

$$
\begin{aligned}
M_{k}[f](s) & =\int_{0}^{1} \mathbf{G}_{2,-s}^{k}[F](x, 0) d x \\
& =\lambda(2-s)^{k} p(s) \int_{0}^{1} F_{2,-s}(x, 0) d x\left(1+O\left(\alpha^{k}\right)\right),
\end{aligned}
$$

uniformément sur un voisinage de $s=0$, et pour $k$ suffisamment grand. Les hypothèses du Théorème 8 de Hwang s'appliquent donc, puisque

$$
U(s)=\log \lambda(2-s) \text { et } V(s)=\log p(s)+\log \left(\int_{0}^{1} F_{2,-s}(x, 0) d x\right)
$$

définissent deux fonctions analytiques sur ce voisinage de $s=0$. Il est clair que $U^{\prime}(0)$ et $U^{\prime \prime}(0)$ ont les formes annoncées dans (70). De plus, Hensley [He] a démontré que $U^{\prime \prime}(0)$ est non nul.

La preuve s'adapte sans problème à l'étude de la série génératrice $M_{k}[f, y]$ des moments de la variable aléatoire $\log \left(Q_{k}+y Q_{k-1}\right)$. Dans ce cas, on a, grâce à (16),

$$
V(s)=\log p(s)+\log \left(\int_{0}^{1} F_{2,-s}(x, y) d x\right) .
$$

L'ensemble des résultats obtenus ici améliore donc les résultats connus tant par l'obtention de la convergence vers la loi normale dans le cadre général d'une densité de référence quelconque, que par l'obtention d'un comportement asymptotique précis où il apparaît une indépendance quantifiée par rapport à la densité de référence.

5. Les opérateurs de Ruelle-Mayer généralisés et l'analyse dynamique de l'algorithme de Gauss. Il s'agit d'étudier maintenant le comportement asymptotique de deux objets : la distribution de probabilités de la variable $L$ représentant le nombre d'itérations et la densité obtenue après $k$ itérations.

Lorsque la densité initiale $f$ est uniforme, le comportement asymptotique de ces deux objets a déjà été étudié [DFV]; il fait intervenir les objets spectraux dominants de l'opérateur $\mathcal{G}_{4}$. La distribution de probabilités du nombre d'itérations est asymptotiquement géométrique, avec une raison-limite égale à $\lambda(4) \approx 0.1994$. La densité des données après $k$ itérations admet une limite pour $k \rightarrow \infty$ proportionnelle à

$$
\int_{-1}^{1}\left(1-w^{2}\right) f_{4}(x+i y w) d w
$$

où $f_{4}$ est la fonction propre dominante de $\mathcal{G}_{4}$. 
Les opérateurs généralisés permettent d'étendre ces résultats quand la densité initiale n'est plus uniforme, en particulier quand elle a une valuation, définie en (18). La Proposition 2 exprime les deux objets étudiés en fonction des itérés $\mathbf{G}_{s, s}^{k}[F](x+i y, x-i y)$ des opérateurs $\mathbf{G}_{s, s}$, ou encore, de manière alternative, en fonction des itérés $\mathcal{H}_{2 s}^{k}[f](x, y)$ des opérateurs $\mathcal{H}_{2 s}$. Rappelons ici que ces derniers opérateurs sont définis, pour un complexe $s$ vérifiant $\Re(s)>1 / 2$, par la relation

$$
\mathcal{H}_{2 s}[f](x, y)=\sum_{m \geq 1} \frac{1}{|m+x+i y|^{2 s}} f\left(\frac{1}{m+x+i y}\right) .
$$

Ces opérateurs ne sont autres que les opérateurs $\mathbf{G}_{s, s}$ qu'on applique à la fonction $F$ de deux variables définie à partir de la densité initiale $f$ par la relation $f(x, y)=F(x+i y, x-i y)$ :

$$
\mathcal{H}_{2 s}[f](x, y)=\mathbf{G}_{s, s}[F](x+i y, x-i y) .
$$

Il est alors équivalent de travailler avec des fonctions $f(x, y)$ qui sont analytiques comme fonction de deux variables réelles $x$ et $y$ ou avec des fonctions $F$ holomorphes par rapport aux deux variables $z=x+i y$ et $\bar{z}=$ $x-i y$. L'opérateur $\mathcal{H}_{s}$ se définit naturellement, pour un nombre complexe $s$ vérifiant $\Re(s)>1 / 2$, sur l'ensemble $C_{\infty}(\mathcal{V})$ des fonctions $g(x, y)$ définies pour $x+i y$ appartenant à $\mathcal{V}$ et qui sont analytiques par rapport aux deux variables $x$ et $y$.

5.1. Comportement asymptotique des itérés de l'opérateur $\mathcal{H}_{s}$. Le résultat suivant adapte les résultats de la section 3 à cet espace de fonctions particulier intervenant dans l'algorithme de Gauss. Il décrit le comportement asymptotique des itérés de l'opérateur $\mathcal{H}_{s}$ en fonction des objets spectraux dominants de l'opérateur $\mathcal{H}_{s}$, qui sont essentiellement les mêmes que ceux de l'opérateur $\mathcal{G}_{s}$.

Proposition 5. Soient un réel $s \geq 1$ et une fonction $g$ analytique (en $x$ et $y$ ), positive sur $\overline{\mathcal{D}}$ et strictement positive sur $\overline{\mathcal{I}}$. Alors on a, pour tout $k$ suffisamment grand et tout $(x, y) \in \mathcal{V}$,

$$
\mathcal{H}_{2 s}^{k}[g](x, y)=\lambda(2 s)^{k} p_{s}[g] F_{s, s}(x+i y, x-i y)\left(1+O\left(\nu(2 s)^{k}\right)\right) .
$$

Ici, $\lambda(2 s)$ est la valeur propre dominante de $\mathcal{G}_{2 s}, F_{s, s}$ est un vecteur propre dominant de $\mathbf{G}_{s, s}$ et $\nu(2 s)$ est le rapport spectral de $\mathbf{G}_{s, s}$. La quantité $p_{s}[g]$ est strictement positive et généralise la notion de projection sur le sousespace dominant. La constante du $O$ dépend de $s$ et de $g$.

Preuve. On applique le Théorème 7 à l'opérateur $\mathbf{G}_{s, s}$, avec un paramètre $s$ réel vérifiant $s \geq 1$ : Pour une fonction $F$ de $B_{\infty}(\mathcal{V})$, il existe une 
constante $A$ dépendant de $F$ pour laquelle on a

$$
\left\|\frac{\mathbf{G}_{s, s}^{k}[F](u, v)}{\lambda(2 s)^{k}}-f_{2 s}^{\star}[f] F_{s, s}(u, v)\right\| \leq A\|F\| \nu(2 s)^{k}
$$

pour tout $k \geq 1$ et pour tout $(u, v) \in \mathcal{V} \times \mathcal{V}$.

On revient alors au problème particulier. A partir d'une fonction $g$ analytique en $(x, y)$ sur $\overline{\mathcal{D}}$, on définit $F$ par $F(x+i y, x-i y)=g(x, y)$. Alors, $F$ est analytique et strictement positive sur la diagonale de $\overline{\mathcal{I}} \times \overline{\mathcal{I}}$, et se prolonge en une fonction analytique sur un voisinage ouvert $\mathcal{W}$ de la diagonale de $]-\varrho, 1+\varrho[\times]-\varrho, 1+\varrho[$. Reprenant un argument similaire à celui utilisé dans la preuve de la relation (68) de la Proposition 4, on montre qu'il existe un entier $k_{0}$ pour lequel

$$
\bigcup_{|h|=k} h(\mathcal{V}) \times h(\mathcal{V}) \subset \mathcal{W} \quad \text { pour tout } k \geq k_{0} .
$$

Ainsi $L:=\mathbf{G}_{s, s}^{k_{0}}[F]$ est une fonction de $B_{\infty}(\mathcal{V})$ et son application diagonale $l=\mathcal{G}_{2 s}^{k_{0}}[f]$, où $f$ est l'application diagonale de $F$, est strictement positive sur $\mathcal{J}$. La projection $f_{2 s}^{\star}[l]$ est donc non nulle. Par ailleurs, la fonction propre

$$
\widehat{f}_{s}(x, y)=F_{s, s}(x+i y, x-i y)=\int_{-1}^{1}\left(1-w^{2}\right)^{(s-1)} f_{2 s}(x+i y w) d w
$$

de $\mathcal{H}_{s}$ est réelle et strictement positive pour $x+i y \in \mathcal{V}$; ceci provient du fait que l'opérateur $\mathbf{G}_{s, s}$ est $u_{0}$-positif, avec une notion plus forte de positivité que dans le cas général ( $s$ et $t$ quelconques de somme réelle) : On considère en effet l'espace $\widetilde{B}_{\infty}(\mathcal{V})$ des fonctions $F$ de $B_{\infty}(\mathcal{V})$ pour lesquelles $F(x+$ $i y, x-i y)$ est réelle pour $x+i y \in \mathcal{V}$, et l'ensemble $K^{\prime \prime}$ formé de la fonction nulle et des fonctions de $\widetilde{B}_{\infty}(\mathcal{V})$ pour lesquelles $F(x+i y, x-i y)$ est positive pour $x+i y \in \mathcal{V}$ mais non identiquement nulle sur $\mathcal{J}$. Le cône $K^{\prime \prime}$ contient la fonction constante $u_{0}$ égale à 1 ; il est propre, reproductif, et d'intérieur non vide. L'opérateur $\mathbf{G}_{s, s}$ laisse stable $\widetilde{B}_{\infty}(\mathcal{V})$. On montre alors par une preuve analogue à celle de la Proposition 3 que pour $s$ réel vérifiant $s \geq 1$, l'opérateur $\mathbf{G}_{s, s}$ restreint à $\widetilde{B}_{\infty}(\mathcal{V})$ est $u_{0}$-positif par rapport au cône $K^{\prime \prime}$. La fonction propre $F_{s, s}$ est donc un élément de l'intérieur du cône $K^{\prime \prime}$ et la fonction $\widehat{f}_{s}(x, y)$ définie en (74) est donc strictement positive pour $x+i y \in \mathcal{V}$.

On a alors, en appliquant le résultat (73) à $L$, et en utilisant la positivité des objets,

$$
\mathbf{G}_{s, s}^{k}[L](x+i y, x-i y)=\lambda(2 s)^{k} f_{2 s}^{\star}[l] F_{s, s}(x+i y, x-i y)\left(1+O\left(\nu(2 s)^{k}\right)\right),
$$

et donc, en remplaçant $L$ par sa valeur et en posant

$$
p_{s}[g]:=\frac{f_{s}^{\star}[l]}{\lambda(2 s)^{k_{0}}},
$$


on obtient, pour tout $k \geq k_{0}$ et tout $x+i y$ de $\mathcal{V}$,

$$
\begin{aligned}
\mathcal{H}_{2 s}^{k}[g](x, y) & =\mathbf{G}_{s, s}^{k}[F](x+i y, x-i y) \\
& =\lambda(2 s)^{k} p_{s}[g] F_{s, s}(x+i y, x-i y)\left(1+O\left(\nu(2 s)^{k}\right)\right),
\end{aligned}
$$

qui est le résultat cherché.

5.2. L'analyse dynamique de l'algorithme de Gauss. Le résultat suivant constitue alors la conclusion du deuxième axe du travail. Il montre que les objets limites décrivant le comportement asymptotique de l'algorithme de Gauss (raison-limite $\varrho$ de la distribution de probabilités, densité limite $F_{\infty}$ ) dépendent de la valuation $r$ de la densité initiale et correspondent exactement aux objets spectraux dominants de l'opérateur $\mathcal{H}_{4+2}$, et s'expriment donc en fonction des objets spectraux dominants de l'opérateur $\mathcal{G}_{4+2 r}$. Les vitesses de convergence vers les objets limite sont exponentielles et font intervenir le rapport spectral $\nu(4+2 r)$.

THÉORÈme 10. On considère une densité initiale $f$ de valuation $r(r>$ $-1)$. Alors :

(i) La distribution de probabilités du nombre d'itérations est asymptotiquement géométrique, avec une raison-limite égale à la valeur propre dominante $\lambda(4+2 r)$ de $\mathcal{G}_{4+2 r}$. La probabilité $\varrho_{k}[f]$ de l'événement $[L \geq k+1]$ vérifie

$$
\varrho_{k}[f]=c_{f} \lambda(4+2 r)^{k}\left(1+O\left(\nu(4+2 r)^{k}\right)\right),
$$

où la constante $c_{f}$ et la constante des $O$ dépendent de $f$.

(ii) La suite $F_{k}[f]$ des densités dans $\mathcal{D}$ après $k$ itérations admet une limite $F_{\infty}[f]$, de valuation $r$, qui s'exprime en fonction du vecteur propre dominant $f_{4+2 r}$ de $\mathcal{G}_{4+2 r}$. Au point $(x, y), F_{\infty}[f]$ est proportionnelle à

$$
|y|^{r} \int_{-1}^{1}\left(1-w^{2}\right)^{r+1} f_{4+2 r}(x+i y w) d w
$$

et le rapport spectral $\nu(4+2 r)$ intervient dans la convergence de $F_{k}[f]$ vers $F_{\infty}[f]:$

$$
\left|F_{k}[f](x, y)-F_{\infty}[f](x, y)\right|=|y|^{r} O\left(\nu(4+2 r)^{k}\right),
$$

où la constante du $O$ dépend de $f$.

Preuve. La preuve est fondée sur la relation (20) de la Proposition 2 et la relation (72) de la Proposition 5.

On retrouve en particulier le résultat de $[\mathrm{DFV}]$ si la densité initiale est uniforme, ce qui correspond à une valuation nulle. Mais, de fait, contrairement à ce qui se passe dans un certain nombre de problèmes en analyse en moyenne d'algorithmes, la densité uniforme n'est pas toujours la plus na- 
turelle dans le contexte étudié. Il y a deux cas intéressants : le cas d'une valuation $r$ tendant vers -1 , et celui d'une valuation $r$ entière.

(i) Si la densité initiale $f$ a une valuation $r$ qui se rapproche de -1 , les données ont alors tendance à se concentrer sur $\mathcal{I}$ et le comportement de l'algorithme de Gauss va se rapprocher du comportement de l'algorithme d'Euclide, et en particulier la raison-limite $\varrho$ tend vers $\lambda(2)=1$. Les opérateurs de Ruelle-Mayer généralisés proposent ainsi un cadre commun où l'on peut analyser les deux algorithmes, celui de Gauss et celui d'Euclide.

Ces opérateurs jouent aussi un rôle central dans l'analyse d'un algorithme qui évalue le signe d'un déterminant $2 \times 2[\mathrm{FV}]$, [Va2], et qui est utilisé en géométrie algorithmique.

(ii) Dans l'algorithme LLL, si l'on part d'un modèle naturel uniforme, les appels à l'algorithme de Gauss ont lieu sur des réseaux de dimension 2 sur lesquels la densité n'est pas uniforme. Plus précisément, le $i$-ème réseau considéré dans un algorithme LLL fonctionnant en dimension $n$ est distribué selon une densité de valuation $n-i-1$. Les opérateurs de RuelleMayer généralisés fournissent aussi un outil qui permet d'aborder l'analyse en moyenne et l'analyse dynamique de l'algorithme LLL.

Conclusion. Le cadre choisi ici est celui des fonctions holomorphes, à une ou à deux variables, et permet d'obtenir à la fois l'existence d'objets spectraux dominants, qui donne la partie principale du comportement asymptotique des objets étudiés, aussi bien que l'évaluation précise du terme d'erreur en fonction des objets spectraux sous-dominants. Comme le fait remarquer le referee de ce papier, on peut se placer dans un cadre plus général de fonctions (seulement Lipschitziennes par exemple ou même à variation bornée) et obtenir également l'existence d'objets spectraux dominants, et donc la partie principale du comportement asymptotique des objets étudiés. Dans ce cadre plus général, il semble alors plus difficile d'obtenir l'évaluation précise du terme d'erreur.

Remerciements. Merci à Philippe Flajolet, Hervé Daudé, Christian Faivre, pour les discussions utiles que j'ai pu avoir avec eux. Tous mes remerciements vont aussi au referee de cet article pour sa lecture attentive et ses propositions constructives.

\section{Bibliographie}

[Ba] K. I. Babenko, On a problem of Gauss, Soviet Math. Dokl. 19 (1978), 136-140.

[Br] A. Broise, Aspects stochastiques de certains systèmes dynamiques : transformations dilatantes de l'intervalle, fractions continues multidimensionnelles, Ph.D. Thesis, Université de Rennes, 1994. 
[DV] H. Daudé and B. Vallée, An upper bound on the average number of iterations of the LLL algorithm, Theoret. Comput. Sci. 123 (1994), 95-115.

[DFV] H. Daudé, P. Flajolet and B. Vallée, An analysis of the Gaussian algorithm for lattice reduction, in: Algorithmic Number Theory (Ithaca, N.Y., 1994), Lecture Notes in Comput. Sci. 877, Springer, 1994, 144-158. Version longue en Rapport de Recherche du Laboratoire GREYC de l'université de Caen, à paraître dans Combinatorics, Probability and Computing.

[Di] J. H. Dixon, The number of steps in the Euclidean algorithm, J. Number Theory 2 (1970), 414-422.

[Fa] C. Faivre, Distribution of Lévy constants for quadratic numbers, Acta Arith. 61 (1992), 13-34.

[FV] P. Flajolet and B. Vallée, Continued fraction algorithms, functional operators and structure constants, Conférence invitée à la 7e Conférence Fibonacci, Graz, juillet 96, Rapport de Recherche INRIA 2931 (juillet 96).

[Ga] C. F. Gauss, Recherches arithmétiques, 1807, réimprimé par Blanchard, Paris, 1953.

[Gr1] A. Grothendieck, Produits tensoriels topologiques et espaces nucléaires, Mem. Amer. Math. Soc. 16 (1955).

[Gr2] —, La théorie de Fredholm, Bull. Soc. Math. France 84 (1956), 319-384.

[Hei] H. Heilbronn, On the average length of a class of continued fractions, in: Number Theory and Analysis, P. Turán (ed.), Plenum, New York, 1969, 87-96.

[He] D. Hensley, The number of steps in the Euclidean algorithm, J. Number Theory 49 (1994), 142-182.

[Hw] H.-K. Hwang, Théorèmes limites pour les structures combinatoires et les fonctions arithmétiques, Ph.D. Thesis, Ecole Polytechnique, Palaiseau, 1994.

[KS] M. Kaib and C. P. Schnorr, A sharp worst-case analysis of the Gaussian lattice basis reduction algorithm for any norm, J. Algorithms, to appear.

[Kr] M. Krasnosel's ki ̌r, Positive Solutions of Operator Equations, Chap. 2, Noordhoff, Groningen, 1964.

[Ku] R. Kuzmin [R. O. Kuz'min], Sur un problème de Gauss, Atti del Congresso internazionale dei matematici, Bologna, 1928, Vol. 6, 83-89.

[Lag] J. C. Lagarias, Worst-case complexity bounds for algorithms in the theory of integral quadratic forms, J. Algorithms 1 (1980), 142-186.

[Lam] D. Lamé, Note sur la limite du nombre de divisions dans la recherche du plus grand commun diviseur entre deux nombres entiers, C. R. Acad. Sci. 19 (1845), 867-870.

[LLL] A. K. Lenstra, H. W. Lenstra and L. Lovász, Factoring polynomials with rational coefficients, Math. Ann. 261 (1982), 513-534.

[Le] P. Lévy, Sur la loi de probabilité dont dépendent les quotients complets et incomplets d'une fraction continue, Bull. Soc. Math. France 57 (1929), 178-194.

[Ma1] D. H. Mayer, Continued fractions and related transformations, in: Ergodic Theory, Symbolic Dynamics and Hyperbolic Spaces, T. Bedford, M. Keane and C. Series (eds.), Oxford University Press, 1991, 175-222.

[Ma2] - On composition operators on Banach spaces of holomorphic functions, J. Funct. Anal. 35 (1980), 191-206.

[Ma3] - Spectral properties of certain composition operators arising in statistical mechanics, Comm. Math. Phys. 68 (1979), 1-8.

[Ma4] - On the thermodynamic formalism for the Gauss map, ibid. 130 (1990), 311333. 
[Ma5] D. H. Mayer, Approach to equilibrium for locally expanding maps in $\mathbf{R}^{k}$, ibid. 95 (1984), 1-15.

[MR] D. Mayer and G. Roepstorff, On the relaxation time of Gauss' continued fraction map. I. The Hilbert space approach, J. Statist. Phys. 47 (1987), 149171, II. The Banach space approach (transfer operator approach), ibid. 50 (1988), 331-344.

[Mi] G. A. Misyavichyus [G. Misevičius], Estimate of the remainder in the limit theorem for the denominators of continued fractions, Litovsk. Mat. Sb. 21(3) (1987), 63-74 (in Russian).

[Mo] T. Morita, Local limit theorem and distribution of periodic orbits of LasotaYorke transformations with infinite Markov partition, J. Math. Soc. Japan 46 (1994), 309-343.

[Ph] W. Philipp, Some metrical theorems in number theory II, Duke Math. J. 37 (1970), 447-488. Errata, ibid., 788.

[RS] A. Rockett and P. Szüsz, Continued Fractions, World Scientific, Singapore, 1992.

[Va1] B. Vallée, Gauss' algorithm revisited, J. Algorithms 12 (1991), 556-572.

[Va2] - Algorithms for computing signs of $2 \times 2$ determinants: dynamics and averagecase algorithms, Les Cahiers Du GREYC, Université de Caen, 1996.

[Wi] E. Wirsing, On the theorem of Gauss-Kusmin-Lévy and a Frobenius-type theorem for function spaces, Acta Arith. 24 (1974), 507-528.

\section{GREYC-URA 1526}

Département d'Informatique

Université de Caen

14032 Caen Cedex, France

E-mail: Brigitte.Vallee@info.unicaen.fr 